\title{
American Society of Regional Anesthesia and Pain Medicine expert panel recommendations on point-of- care ultrasound education and training for regional anesthesiologists and pain physicians-part I: clinical indications
}

\author{
Stephen C Haskins 주 1,2 Yuriy Bronshteyn, ${ }^{3}$ Anahi Perlas 다, ${ }^{4}$ \\ Kariem El-Boghdadly (1) ${ }_{1}^{5}$ Joshua Zimmerman, ${ }^{6}$ Marcos Silva, ${ }_{1}^{4}$ Karen Boretsky, ${ }^{7}$ \\ Vincent Chan ${ }_{1}^{4}$ Richelle Kruisselbrink, ${ }^{8}$ Melissa Byrne, ${ }^{9}$ Nadia Hernandez, ${ }^{10}$ \\ Jan Boublik, ${ }^{11}$ William Clark Manson (10, ${ }^{12}$ Rosemary Hogg ${ }^{13}$ Jonathan N Wilkinson, ${ }^{14}$ \\ Hari Kalagara, ${ }^{15}$ Jemiel Nejim, ${ }^{1,2}$ Davinder Ramsingh $\left({ }^{10},{ }^{16}\right.$ Hariharan Shankar, ${ }_{1}^{17}$ \\ Antoun Nader, ${ }^{18}$ Dmitri Souza, ${ }_{19}^{19}$ Samer Narouze (1) ${ }^{19}$
}

For numbered affiliations see end of article.

Correspondence to Dr Stephen C Haskins, Anesthesiology, Hospital for Special Surgery, New York, NY 10021, USA; haskinss@hss.edu

Received 29 January 2021 Accepted 4 February 2021

\section{Linked}

- http://dx.doi.org/10.1136/ rapm-2021-102561

Check for updates

(C) American Society of Regional Anesthesia \& Pain Medicine 2021. No commercial re-use. See rights and permissions. Published by BMJ.

To cite: Haskins SC, Bronshteyn Y, Perlas A, et al. Reg Anesth Pain Med Epub ahead of print: [please include Day Month Year]. doi:10.1136/rapm-2021102560

\section{ABSTRACT}

Point-of-care ultrasound (POCUS) is a critical skill for all regional anesthesiologists and pain physicians to help diagnose relevant complications related to routine practice and guide perioperative management. In an effort to inform the regional anesthesia and pain community as well as address a need for structured education and training, the American Society of Regional Anesthesia and Pain Medicine (ASRA) commissioned this narrative review to provide recommendations for POCUS. The guidelines were written by content and educational experts and approved by the Guidelines Committee and the Board of Directors of the ASRA. In part I of this two-part series, clinical indications for POCUS in the perioperative and chronic pain setting are described. The clinical review addresses airway ultrasound, lung ultrasound, gastric ultrasound, the focus assessment with sonography for trauma examination and focused cardiac ultrasound for the regional anesthesiologist and pain physician. It also provides foundational knowledge regarding ultrasound physics, discusses the impact of handheld devices and finally, offers insight into the role of POCUS in the pediatric population.

\section{INTRODUCTION}

Point-of-care ultrasound (POCUS) has become a powerful tool at the bedside because of the potential for improving patient management. Modern ultrasound (US) equipment provides excellent image quality, portability, simplicity and availability in various clinical settings. Although ultrasound and echocardiography have historically been within the purview of radiologists and cardiologists, the emergence of POCUS as a bedside tool to supplement the physical examination has resulted in tremendous expansion into other subspecialties. Although there is overlap, there are unique subspecialty clinical applications. For example, ultrasound visualization of peripheral nerves for procedural purposes has a significant benefit to regional anesthesiologists and chronic pain physicians despite having separate indications in radiology. ${ }^{1-3}$ It is this diversity in clinical ultrasound practice among subspecialties that underlies the American Medical Association's recommendation that 'policy on ultrasound imaging... (and) criteria for granting ultrasound privileges (be) based upon background and training for the use of ultrasound technology and strongly recommends that these criteria are in accordance with recommended training and education standards developed by each physician's respective specialty'. ${ }^{4}$ Based on these recommendations, there exist guidelines for training, competency and credentialing in POCUS in emergency medicine ${ }^{5}$ and critical care medicine, ${ }^{67}$ and there is currently a work product for POCUS available through the American Society of Anesthesiologists (ASA). ${ }^{8}$ Despite the availability of significant resources in the form of numerous live courses ${ }^{9}$ and an educational review series on POCUS, ${ }^{10-15}$ no specific guidelines or recommendations exist on the use of POCUS for regional anesthesiologists and pain physicians. Given ASRA's early leadership in providing recommendations for procedural-based bedside ultrasound in the form of ultrasound-guided regional anesthesia and ultrasound-guided interventional pain procedures, there was a call to address the need for formal POCUS recommendations. ${ }^{23}$

The American Society of Regional Anesthesia and Pain Medicine (ASRA) Guidelines Committee appointed a task force to develop recommendations for the clinical indications for POCUS as a foundation for education and training in POCUS. The task force aims to provide alignment between regional anesthesiologists and acute and chronic pain physicians for training recommendations in the perioperative and/or chronic pain setting. The 
recommendations are based on the current best evidence and will consider each clinical setting's commonalities and uniqueness.

In part I of a two-part review article series, we will discuss the clinical indications for POCUS for the regional anesthesiologist and pain physician. The skills discussed are airway ultrasound, lung ultrasound (LUS), gastric ultrasound, the focused assessment with sonography for trauma (FAST) examination and focused cardiac ultrasound (FoCUS). The authors acknowledge that musculoskeletal ultrasound and neurosonography are other emerging diagnostic skills but are outside the scope of this POCUS article.

We also provide foundational knowledge regarding ultrasound physics, the impact of handheld devices and finally, insight into the role of POCUS in the pediatric population. ${ }^{15}$ Ultimately, these task force recommendations will outline the clinical indications of POCUS and lay the foundation for defining criteria for education, training, competency and credentialing for the regional anesthesia and pain physician.

\section{METHODS}

An expert panel was assembled for this project based on the recommendations from the ASRA's Guidelines Committee and Board of Directors. This panel consists of national and international experts in POCUS. Specifically, the qualifications for panel selection involved multiple publications in POCUS, leadership through participation in and/or development of POCUS education and expertise and leadership in regional anesthesia and pain medicine to ensure all topics are accurately covered and appropriate for education and training recommendations. To define areas of competency, a systematic literature search (MEDLINE, PubMed and Ovid) was performed, looking at clinical indications and utilization of POCUS for anesthesiologists, regional anesthesiologists and pain physicians. Findings were not filtered by risk of bias or Grading of Recommendations, Assessment, Development and Evaluations (GRADE) of evidence. When appropriate, recommendations were drawn from other subspecialties such as critical care, cardiology, emergency medicine, surgery and radiology. Keywords used included: point-of-care ultrasound, ultrasound, regional anesthesiology, perioperative point-of-care ultrasound, chronic pain, acute pain, ultrasonography/standards, clinical competency/standards, ultrasound physics, curriculum, education, training, barriers to education, barriers to training, airway ultrasound, lung ultrasound, pulmonary ultrasound, focused cardiac ultrasound, gastric ultrasound, pediatric point-of-care ultrasound and handheld ultrasound. Based on these relevant clinical skills (airway ultrasound, lung ultrasound, focused cardiac ultrasound, gastric ultrasound, abdominal/pelvic ultrasound), corresponding groups were tasked with describing educational goals and requirements for competency for regional anesthesiologists and pain physicians. The writing process was then conducted in leaderfacilitated groups. Contributing authors were granted access to review the document in its entirety and gave final approval to the recommendations.

\section{Ultrasound physics and equipment requirements for POCUS}

Ultrasound imaging is a user-dependent tool that requires knowledge of the fundamental principles of ultrasound and the technical skills for acquisition, optimization and accurate interpretation of images. ${ }^{16}$ Most regional anesthesiologists and pain physicians are familiar with linear and curvilinear transducers for ultrasound-guided procedures and the general principles of ultrasound image generation, common artifacts and the

\begin{tabular}{llll}
\multicolumn{3}{l}{ Table 1} & Knowledge of ultrasound physics and equipment ${ }^{21}$ \\
\hline $\begin{array}{l}\text { Ultrasound } \\
\text { physics }\end{array}$ & Artifacts & Equipment & $\begin{array}{l}\text { Instrument } \\
\text { functions }\end{array}$ \\
\hline $\begin{array}{l}\text { Amplitude } \\
\text { Wavelength }\end{array}$ & Axial & Piezoelectric & Gain \\
Frequency & Comet tail & transducers & Time gain \\
Intensity & Enhancement & Linear array & compensation \\
Absorption & Reverberation & Convex array & Preset \\
Impedance & Mirroring & Depth \\
Attenuation & Lateral & Phased array & Focus \\
Reflection & Side lobes & $2-7.5 \mathrm{MHz}$ & Motion mode \\
Refraction & Refraction & Vector array (linear & Color Doppler \\
Scattering & Equipment & and phased) & Power Doppler \\
Harmonics & Range ambiguity & $1.25-4.5 \mathrm{MHz}$ & Pulse wave Doppler \\
Axial resolution & Aliasing & Capacitive & Spectral Doppler \\
Lateral resolution & Ghosting & transducers & Harmonics \\
& & CMUT & Saving images \\
& & CPUT & Clip function \\
\hline
\end{tabular}

CMUT, capacitive micromachined ultrasound transducer; CPUT, capacitive piezoelectric ultrasound transducers.

sonoanatomy of neurovascular structures. To perform an organbased ultrasound, such as echocardiography, clinicians will need to familiarize themselves with lower frequency transducers, such as the phased array, as well as the wide dynamic range capacitive micromachined ultrasound transducers (CMUTs). The artifacts associated with phased array processing, such as ghosting, and Doppler measurements, such as aliasing, are unique and require advanced understanding. ${ }^{17} 18$

Medical ultrasound is mechanical energy in the form of high frequency $(>20 \mathrm{kHz})$ sound waves emitted from a piezoelectric transducer or a CMUT. The majority of the waves pass through tissue with different densities and resistance to sound (acoustic impedance), and a small fraction is reflected toward the transducer. Returned ultrasound signals alter the shape of the piezoelectric crystals or flexible plates of the CMUT, creating an electrical current proportional to the signal's strength, which is then processed to form a two-dimensional image. ${ }^{19}$ Concepts such as reflection, refraction, scattering and attenuation play an essential role in ultrasound image interpretation; however, given the scope of this article, we suggest learners reference other fundamentals of ultrasound text to explore these topics further.

Ultrasound artifacts play an important role in POCUS imaging and interpretation. With POCUS, navigating through acoustic barriers such as aerated lungs and the bony framework of the chest wall results in artifacts that can either contribute to misdiagnosis or be used as diagnostic aids. ${ }^{20}$ For example, A-lines and B-lines, discussed in detail in the LUS section, can be used to guide management in focused LUS. See table 1 for a list of common ultrasound artifacts.

For POCUS, transducer selection ensures optimal axial and lateral resolution. High-frequency linear (HFL) transducers provide a rectangular image; however, due to increased attenuation, imaging depth is limited. Therefore, HFL probes are ideal for superficial structures such as the pleura and the airway. Convex (curvilinear) sequential array transducers are arranged in a curved fashion producing a fan-shaped image. Convex transducers have a lower frequency and thereby provide improved image resolution of deeper structures (penetration), a wider image in the far field and are best for imaging intraperitoneal and retroperitoneal organs. For intrathoracic structures such as the heart and large vessels, low-frequency phased array transducers with a small footprint that use electronic field steering to image beyond acoustic barriers, such as ribs, produce 
a cone-shaped image of the heart. When comparing capacitive transducers (CMUT) to traditional piezoelectric transducers, the CMUT transducer's advantages are a larger bandwidth and lower production cost. ${ }^{21}$ CMUT arrays can be manufactured with different operating frequencies and geometries from a single silicon wafer, allowing full-body imaging with a single transducer. $^{22}$ The disadvantage is that CMUT technology is relatively new compared with piezoelectric transducers; therefore, the imaging quality and applications do not match traditional transducers.

\section{CLINICAL INDICATIONS AND EVIDENCE FOR POCUS}

We will focus on the clinical indications and evidence for airway ultrasound, LUS, gastric ultrasound, the FAST examination and FoCUS for the regional anesthesiologist and pain physician.

Although the clinical benefits of POCUS have been widely advocated by many medical specialties, ${ }^{6}{ }^{23-27}$ the apparent positive impact of POCUS on patient outcomes has not consistently been demonstrated in a randomized, controlled trial for many reasons. ${ }^{28} 29$ First, POCUS is used as a diagnostic or monitoring tool. It is not a procedural intervention that can be easily randomized and directly attributed to improved patient outcomes. Second, POCUS is only one part of the complex sequence of medical care. As such, it is challenging to show that a single intervention can either lead to a meaningful outcome or overtreatment, overdiagnosis or potential iatrogenic injury. Finally, POCUS findings as an extension of the physical examination are heavily dependent on proper education and training, maintenance of competency, personal skills and experience and individual interpretation of the operator. With that said, what follows is the current best evidence supporting the clinical indications for POCUS.

\section{Airway ultrasound}

Point-of-care airway ultrasound can aid in the assessment and planning of perioperative or periprocedural airway management. Airway US can be used to predict potential difficult airway management. The measurement of the anterior neck has shown that the distance from the skin to the hyoid bone is highly predictive of difficult mask ventilation and intubation. ${ }^{30}$ In a patient with a concern for a 'cannot intubate, cannot ventilate' scenario, front of neck airway sonographic identification of the cricothyroid membrane is more accurate than palpation alone ${ }^{31-34}$ and more rapid for emergency airway access. ${ }^{35}$ Of note, the cricothyroid membrane is highly dependent on neck position; therefore, one must maintain the same position or (following any movement of the neck) rescan to confirm location before attempting an emergency airway.

Airway ultrasound assessment of the cricoid cartilage compared with palpation has improved accuracy, facilitating the correct application of cricoid pressure. ${ }^{3738}$ Additionally, the efficacy of cricoid pressure can be assessed by visualization of esophageal compression.

Confirmation of endotracheal intubation compared with endobronchial intubation with airway ultrasound was more sensitive and specific than chest auscultation when tracheal cuff dilation and bilateral pleural sliding was seen. ${ }^{39} 40$ In the event of decreased or absent end-tidal carbon dioxide, as seen in cardiac arrest or other low output states, lung sliding can be used as a surrogate for endotracheal tube confirmation. ${ }^{41}$ And finally, it can facilitate nasogastric tube placement. ${ }^{30-40}$ Multiple studies have shown the benefit of ultrasound imaging in the correct placement of nasogastric tubes. ${ }^{42-44}$ See figure 1 for an example of airway ultrasound probe placement, anatomy and sonoanatomy.

There are advanced procedural applications of point-ofcare airway ultrasound that we will briefly mention as they are beyond this document's scope. Ultrasound-guided nerve blocks for airway anesthetization should be reserved for those with expertise in their performance. ${ }^{45}$ Landmark-based glossopharyngeal and superior laryngeal nerve blocks have been associated with higher plasma concentrations of local anesthesia, a higher incidence of local anesthetic systemic toxicity (LAST) and lower patient comfort than less invasive techniques. ${ }^{45}$ While ultrasound-guided techniques may provide more favorable results over traditional landmark-based approaches, the current evidence is insufficient to recommend them over non-invasive mucosal topicalization.

Additionally, a thorough ultrasound assessment of the neck for pathologies such as esophageal (Zenker) diverticulum or an aberrant vertebral artery before performing a stellate ganglion block can avoid potential complications. ${ }^{46} 47$

The Indication, Acquisition, Interpretation, and Medical Decision-Making (I-AIM) Framework is a standardized, step-bystep guide for clinicians learning a new POCUS skill to identify the appropriate clinical scenarios where it can be used. ${ }^{48}$ As airway ultrasound is an emerging skill for the regional anesthesiologists and pain physicians, table 2 provides a review of clinical case examples in the I-AIM Framework to facilitate knowledge comprehension, integration and translation into the clinical arena.

\section{Lung ultrasound}

Point-of-care LUS can be used to assess an acute or critically ill patient with respiratory distress as an extension to physical examination. ${ }^{50}$ LUS has superior diagnostic accuracy (both sensitivity and specificity) over many traditional imaging. Furthermore, LUS is faster to execute and allows repeated dynamic bedside assessment to monitor disease progression or regression (response to treatment) without radiation exposure.

Overall, LUS excels in its accuracy in detecting lung pathologies such as pneumothorax and pleural effusion (table 3). Additionally, LUS has the advantage of differentiating pleural effusion from lung consolidation, which is not always possible with a chest radiograph. ${ }^{51}$

Two large meta-analyses found the sensitivity and specificity of LUS for diagnosis of pneumonia to range between $85 \%-93 \%$ and $72 \%-93 \%$, respectively. ${ }^{52}$ For diagnosis of lung contusion, LUS has a higher degree of diagnostic accuracy than chest radiography with greater sensitivity (95\% vs 27\%) but similar specificity (96\% vs $100 \%) .{ }^{5455}$ LUS is also superior to auscultation in confirming tracheal versus bronchial intubations with higher sensitivity (93\% vs 66\%) and higher specificity $(96 \%$ vs $59 \%) .{ }^{40}$ A recent meta-analysis suggests a higher sensitivity (94\%) and specificity (92.4\%) with LUS to diagnose heart failure than routine clinical workup, including chest radiography and natriuretic peptides. ${ }^{56}$ B-lines are consistently accurate in the diagnosis and monitoring of pulmonary edema and other lung conditions such as acute respiratory distress syndrome, lung infection, ${ }^{57}$ connective-tissue disorders and lung fibrosis. ${ }^{58}$

LUS has been used for decades as a means to assess diaphragmatic function. ${ }^{59}$ The most common methods image the right and left hemidiaphragm by using the liver and spleen as acoustic windows to record diaphragmatic movement in motion mode. The subcostal method, however, can be challenging, particularly on patients with large body habitus. An alternate approach is 


\section{A. Probe Position}
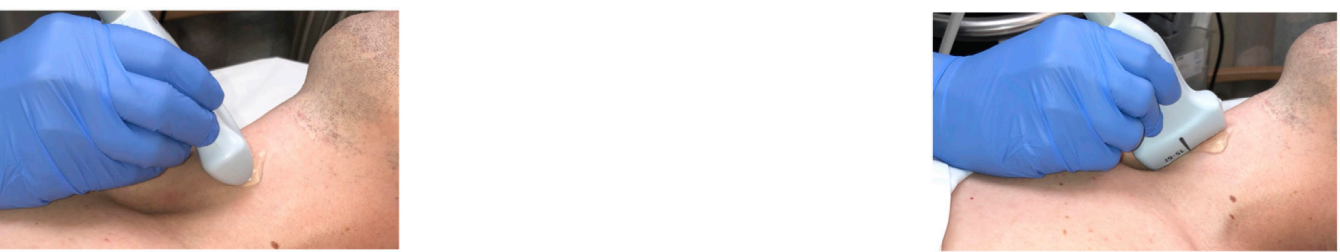

\section{B. Anatomy/Sonoanatomy}
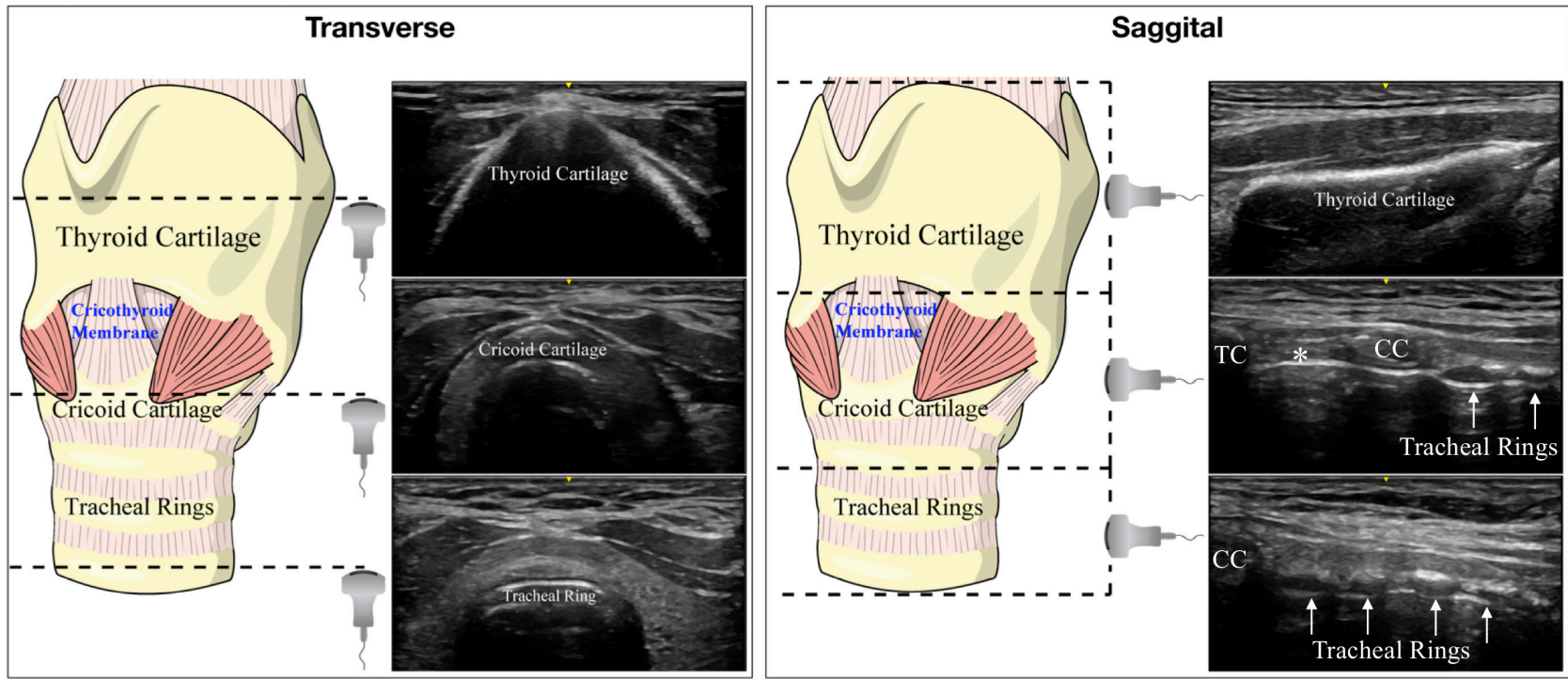

Figure 1 Airway ultrasound (US) probe placement, anatomy and sonoanatomy. (A) Probe placement for airway US scanning. (B) Anatomy of the thyroid cartilage, cricoid cartilage and tracheal rings with corresponding sonoanatomy in transverse and sagittal planes. CC, cricoid cartilage; TC, tracheal cartilage. * Cricothyroid membrane.

to assess diaphragmatic function at the zone of apposition by observing diaphragm muscle thickening during the respiratory cycle. ${ }^{60} 61$ The zone of apposition is defined as the area of the diaphragm close to the lower rib cage where the diaphragm separates from the rib cage.

LUS is based predominantly on the interpretation of artifacts derived by air/tissue interface (eg, pleural line) and real anatomical images in the absence of air/tissue interface (eg, effusion and consolidation). Several lung artifacts (A-lines and B-lines) and signs (lung sliding and pulse) are commonly observed and are highlighted in figure $2 .{ }^{11}$ Interpretation of lung and pleural artifacts or 'signs' has led to the development of standardized protocols to evaluate the lung. These diagnostic protocols follow a step-by-step approach based on particular US profiles. ${ }^{62}$ In the bedside LUS in emergency protocol, ${ }^{62}$ profiles have been designed to assess pneumonia, congestive heart failure, chronic obstructive pulmonary disease (COPD), asthma, pulmonary embolism (PE) and pneumothorax with an accuracy $>90 \%$. The fluid administration limited by lung sonography protocol ${ }^{63}$ sequentially rules out an obstructive, cardiogenic, hypovolemic and distributive (usually septic) shock. ${ }^{64}$

LUS is a valuable tool for diagnosing and monitoring a variety of pulmonary and pleural complications after regional anesthesia and interventional pain procedures. Pneumothorax ${ }^{54}$ has been reported as a potential complication of regional anesthesia procedures such as interscalene ${ }^{65}$ and supraclavicular ${ }^{66}$ brachial plexus block, erector spinae plane block, ${ }^{67}$ thoracic paravertebral block ${ }^{68}$ and pain procedures such as trigger point injection, ${ }^{69}$ intercostal nerve block, ${ }^{70}$ thoracic facet joint injection, radio-frequency lesioning ${ }^{71}$ and celiac plexus block. ${ }^{72}$ Respiratory symptoms may develop within minutes following these procedures but more commonly develop over several hours. Hemidiaphragmatic paresis is a known complication of above the clavicle brachial plexus blocks (interscalene and supraclavicular) due to a secondary phrenic nerve block. ${ }^{59} 73$ LUS can be used to help determine pre/post block diaphragmatic function, monitor the return of diaphragmatic function and differentiate between a block complication versus another potential cause of respiratory insufficiency. Also, pleural effusion and hemothorax have been described after supraclavicular block ${ }^{74}$ and thoracic epidural $^{75}$ and during interscalene brachial plexus infusion. ${ }^{7677}$ Diagnostic LUS can also guide management in acute respiratory failure ${ }^{78}$ circulatory shock and cardiac arrest states, ${ }^{79}$ interstitial syndrome, ${ }^{80}$ lung consolidation, ${ }^{52}$ acute decompensated heart failure $^{81}$ and endobronchial intubation. ${ }^{40} 82$

\section{FAST examination}

The FAST is a well-validated POCUS examination with broad applications in the fields of regional anesthesiology and chronic pain medicine. ${ }^{83}$ See figure 3 for the four FAST views-subcostal, right upper quadrant, left upper quadrant (LUQ) and pelvic views.

Initially, the FAST examination was intended to rapidly identify trauma patients who need surgical intervention. ${ }^{82}$ In the perioperative setting, the indications are an ongoing assessment of trauma patients, critical patients recovering postoperatively, hip arthroscopy patients with severe postoperative pain or showing signs of hemodynamical instability, patients with ascites, patients with peritoneal dialysis and patients following any abdominal surgery. ${ }^{14}{ }^{84}$ Additionally, there is a role in gynecologic and obstetrics procedures in the form of 
Table 2 Examples of cases in airway POCUS following an I-AIM Framework

\begin{tabular}{|c|c|c|c|c|}
\hline Clinical scenario & Indications & Image acquisition & Image interpretation & Medical decision-making \\
\hline Awake cricothyroidotomy & $\begin{array}{l}\text { Location of the cricothyroid } \\
\text { membrane } \\
\text { Location of the cricoid cartilage } \\
\text { Location of tracheal rings }\end{array}$ & $\begin{array}{l}\text { Patient supine with the neck } \\
\text { extended } \\
\text { Linear high-frequency transducer } \\
\text { Scan in transverse and sagittal } \\
\text { planes } \\
\text { Identify: } \\
\text { Thyroid cartilage } \\
\text { Cricothyroid membrane } \\
\text { Cricoid cartilage } \\
\text { Tracheal rings } \\
\text { Thyroid gland } \\
\text { Surrounding vasculature }\end{array}$ & $\begin{array}{l}\text { Localization of cephalocaudal } \\
\text { level, mid-point and size of the } \\
\text { cricothyroid membrane } \\
\text { Localization of the cephalocaudal } \\
\text { level and mid-point of the cricoid } \\
\text { cartilage } \\
\text { Localization of the cephalocaudal } \\
\text { level, mid-point and number of } \\
\text { tracheal rings }\end{array}$ & $\begin{array}{l}\text { Determine and mark the skin for } \\
\text { the correct location of cricothyroid } \\
\text { membrane puncture } \\
\text { Determine and mark the skin for } \\
\text { the correct location of cricoid force } \\
\text { (pressure) }\end{array}$ \\
\hline $\begin{array}{l}\text { Confirmation of correct endotracheal } \\
\text { tube placement }\end{array}$ & $\begin{array}{l}\text { Unclear endotracheal } \\
\text { intubation } \\
\text { Real-time confirmation of } \\
\text { endotracheal intubation } \\
\text { Rule out esophageal intubation }\end{array}$ & $\begin{array}{l}\text { Patient supine } \\
\text { Linear high-frequency transducer } \\
\text { Scan in transverse and sagittal } \\
\text { planes } \\
\text { Identify: } \\
\text { Tracheal rings } \\
\text { Esophagus } \\
\text { Thyroid gland } \\
\text { Surrounding vasculature } \\
\text { Bilateral lung sliding }\end{array}$ & $\begin{array}{l}\text { Verification of endotracheal tube } \\
\text { position (double-bullet sign) } \\
\text { Diagnosis of endobronchial } \\
\text { intubation (double-bullet sign and } \\
\text { unilateral lung sliding (left) } \\
\text { Verification of esophageal } \\
\text { position } \\
\text { (double-tract sign) }\end{array}$ & $\begin{array}{l}\text { Determine the safety of utilization } \\
\text { of a tracheal tube for ventilation } \\
\text { Reintubation if in the esophagus }\end{array}$ \\
\hline $\begin{array}{l}\text { Confirmation of orogastric or } \\
\text { nasogastric tube placement }\end{array}$ & $\begin{array}{l}\text { Orogastric or nasogastric tube } \\
\text { placement without aspiration } \\
\text { of gastric content by suction }\end{array}$ & $\begin{array}{l}\text { Patient supine } \\
\text { Linear high-frequency transducer, } \\
\text { potentially curvilinear probe } \\
\text { for verification of orogastric or } \\
\text { nasogastric tube in the stomach } \\
\text { Scan in transverse and sagittal } \\
\text { planes } \\
\text { Identify: } \\
\text { Tracheal rings } \\
\text { Esophagus } \\
\text { Thyroid gland } \\
\text { Surrounding vasculature }\end{array}$ & $\begin{array}{l}\text { Verification of small, round } \\
\text { structure in esophagus or } \\
\text { stomach }\end{array}$ & $\begin{array}{l}\text { Determine the safety of use of } \\
\text { orogastric or nasogastric tube } \\
\text { Replacement/adjustment of the } \\
\text { position of orogastric or nasogastric } \\
\text { tube }\end{array}$ \\
\hline
\end{tabular}

I-AIM, Indication, Acquisition, Interpretation, and Medical decision-making; POCUS, Point-of-care ultrasound.

the focused assessment with sonography for obstetrics examination. ${ }^{85} 86$ A positive examination indicates that the patient has at least $300-500 \mathrm{~mL}$ of free fluid in the peritoneal space. ${ }^{87}$ This amount of fluid following abdominal surgery is rarely benign and almost always indicates ongoing hemorrhage. An exception to this situation is intra-abdominal fluid extravasation (IAFE) following hip arthroscopy. IAFE occurs in up to $15 \%$ of hip arthroscopy cases and is highly associated with increased pain. ${ }^{84}$

Chronic pain procedures, such as celiac plexus block, have a risk for bleeding and intra-abdominal hemorrhage. ${ }^{88}$ Additionally, patients will rarely have a positive FAST examination except in cases of chronic pathology. It is essential to recognize that cirrhotic patients will commonly accumulate enough ascites to produce a positive FAST examination. The same applies to endstage renal patients treated with peritoneal dialysis.

\begin{tabular}{lll}
\hline $\begin{array}{l}\text { Table } 3 \\
\text { CXR }\end{array}$ & Comparison of sensitivity and specificity between LUS versus \\
\hline & Sensitivity & Specificity \\
\hline Pneumothorax $^{5455}$ & LUS-88\% & LUS-100\% \\
& CXR-52\% & CXR-99\% \\
Pleural effusion $^{183}$ & LUS-94\% & LUS-98\% \\
& CXR-51\% & CXR-91\% \\
\hline
\end{tabular}

CXR, chest $X$-ray; LUS, lung ultrasound.

\section{Gastric ultrasound}

Gastric POCUS is used to evaluate stomach contents as they relate to aspiration risk. ${ }^{13} 89$ Pulmonary aspiration of gastric contents has significant perioperative morbidity and mortality. ${ }^{90}$ Regional anesthesia and pain management procedures are commonly performed under various degrees of sedation. Chronic pain patients frequently require sedation because of increased sensitivity to needling, opioid-induced hyperalgesia and anxiety. Additionally, deep sedation can be crucial for interventions where patient movement during the procedure may lead to devastating complications, including, for example, inadvertent administration of brain stem injection with trigeminal nerve block or radiofrequency ablation, or pneumothorax with intercostal, or paravertebral nerve blocks. ${ }^{91}$

The ASA's recommendations for nil per os (NPO) status are routinely observed in anesthesia and pain medicine practice. ${ }^{92}$ However, the guidelines are for healthy patients undergoing elective procedures and does not apply to the many complex patients receiving anesthesia or sedation. Opioid dependency, labor, diabetic gastroparesis, advanced renal or liver dysfunction, neuromuscular disorders, ileus, trauma or urgent surgery may prolong gastric emptying, leading to a 'full stomach' despite prolonged periods of fasting. ${ }^{93}$ Morbidly obese, pregnant and pediatric patients may also be at increased risk of aspiration. ${ }^{93}$ Additionally, the prandial status may be difficult to assess in patients with a language barrier, altered cognition (such as delirium or dementia) and inconsistent clinical history. 


\section{A. Probe Position}
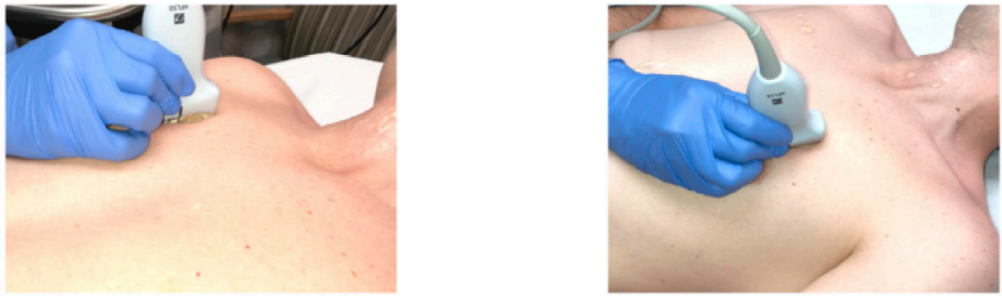

\section{B. Normal Lung}
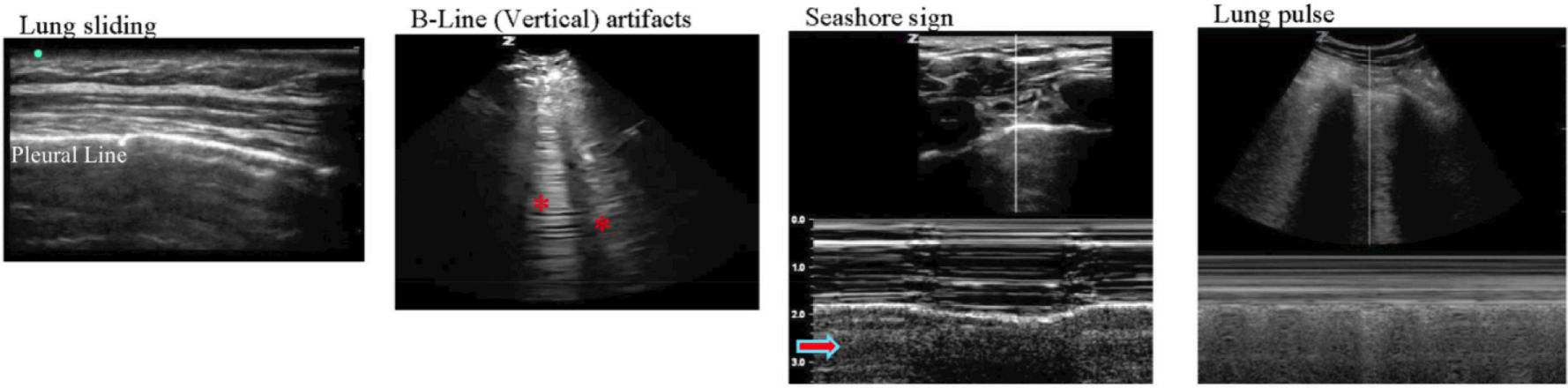

\section{Pneumothorax}
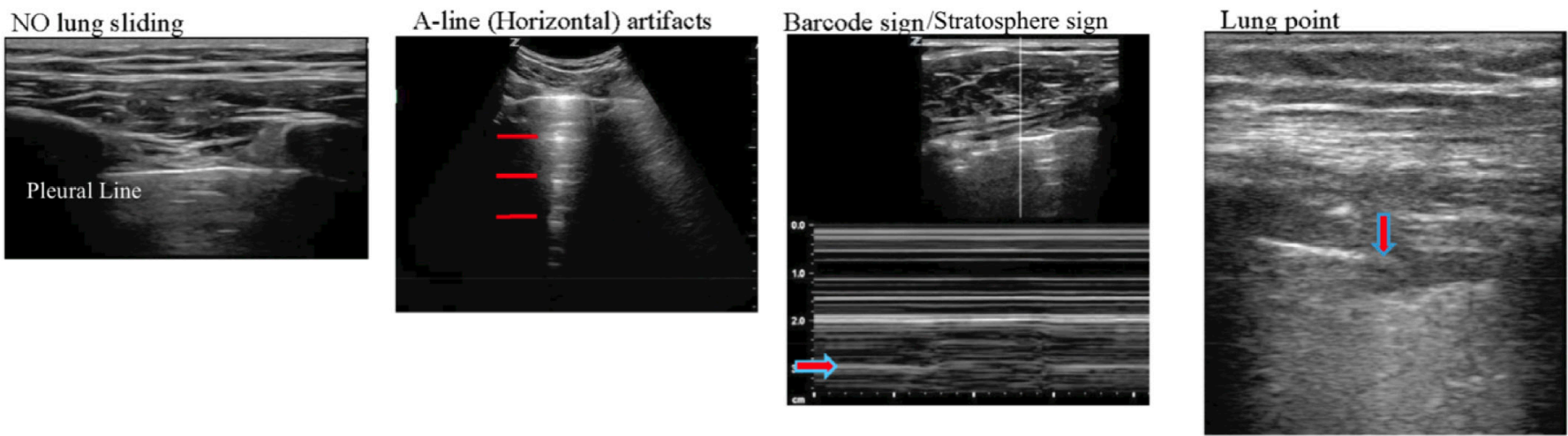

Figure 2 Lung ultrasound (LUS) with normal findings and pathology to rule out and diagnose a pneumothorax. (A) Probe placement for LUS scanning. (B) Normal lung sonoanatomy to rule out a pneumothorax, including lung sliding, B-lines (red asterisks) vertical reverberation artifacts, the seashore sign with arrow pointing to the 'sandy beach' visible with normal lung sliding and lung pulse with asterisk showing intermittent movement synchronous with the cardiac rhythm. (C) Sonoanatomy with LUS to diagnose a pneumothorax, including absence of lung sliding, A-lines (red lines) horizontal artifact pattern seen with absence of lung sliding, a barcode sign or stratosphere sign with the arrow pointing to the pattern of parallel horizontal lines continuing beyond the pleural line visible with lack of movement and a lung point.

Therefore, gastric US should be considered before sedation to verify gastric content for patients with these conditions or when fasting status is unclear. ${ }^{95}$

Gastric POCUS is most useful when there is clinical uncertainty regarding the status of stomach contents, in other words, when the pretest probability of a 'full stomach' is close to 50\%. ${ }^{13} 9697$ In a prospective study with a simulated clinical scenario with a pretest probability of 'full stomach' of $50 \%$, a positive gastric ultrasound examination increased the post-test probability of a full stomach to over $95 \%$, and a negative test decreased the post-test probability to $0.1 \% .^{98}$ Additionally, bedside gastric ultrasound has been shown to change anesthetic management in two-thirds of patients who have not followed fasting instructions before elective surgery ${ }^{99100}$ and in pediatric patients undergoing urgent surgery. 101102

Gastric ultrasound has been studied in adult, ${ }^{103-105}$ pediatric, ${ }^{101106107}$ obstetric $^{108109}$ and morbidly obese ${ }^{110111}$ patients.
A systematic assessment of the gastric antrum provides information about the entire organ's contents (figure 4). ${ }^{103} 104112113$ Based on qualitative and quantitative findings, the results of a gastric ultrasound examination may be summarized in a binary manner as an 'empty' or a 'full' stomach. ${ }^{13} 899697103104114$ An 'empty' stomach has no appreciable content or there is a low volume $(\leq 1.5 \mathrm{~mL} / \mathrm{kg})$ of hypoechoic fluid consistent with baseline gastric secretions. 13899697103104114 A 'full' or 'not empty' stomach has evidence of solid particulate content (mixed echogenicity), thick (hyperechoic) fluid or a volume of clear fluid in excess of normal baseline gastric secretions $(>1.5 \mathrm{~mL} / \mathrm{kg})$ (figure 5). 139697103104114

Patient position affects antral size as the stomach's contents pool in the most dependent areas. A more accurate evaluation of the antrum and gastric content is obtained in the right lateral decubitus (RLD) or the semirecumbent position with the head elevated at $45^{\circ}$, as the supine position can underestimate gastric 
A. Probe Position

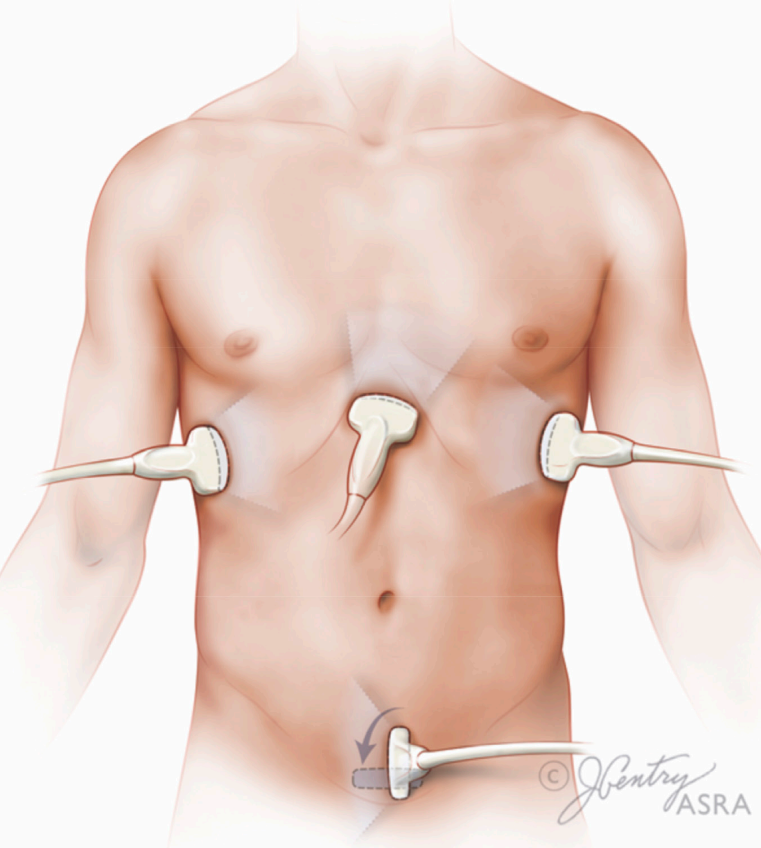

B. Sonoanatomy

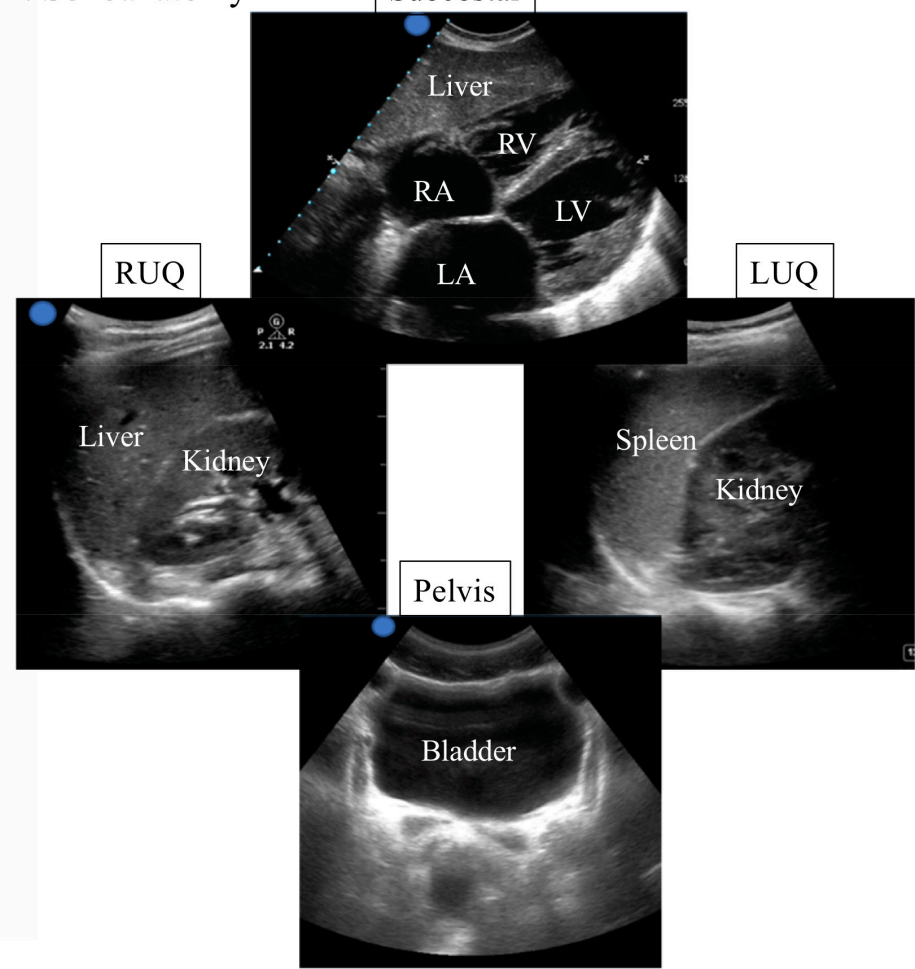

Figure 3 FAST examination probe placement and corresponding sonoanatomy. (A) Probe placement. (B) Sonoanatomy for FAST views including subcostal four-chamber view, RUQ (Morison's pouch/hepatorenal space), LUQ (perisplenic space) and pelvic view (transverse view). FAST, focused assessment with sonography for trauma; LA, left atrium; LUQ, left upper quadrant; LV, left ventricle; RA, right atrium; RUQ, right upper quadrant; RV, right ventricle.

content. ${ }^{103104112115}$ Given these positional differences, a semiquantitative three-point grading system can be used to assess the presence or absence of clear fluid in the supine and/or RLD positions. ${ }^{104112}$ In a grade 0 antrum (empty stomach), fluid is absent in both positions. A grade 1 antrum only has visible fluid in the RLD position, consistent with baseline gastric secretions. Grade 0 and 1 antrums are commonly seen in the fasting state. Alternatively, a grade 2 antrum appears distended with clear fluid in both the supine and RLD positions, and it correlates with gastric volumes $>100 \mathrm{~mL} .{ }^{104} 112 \mathrm{~A}$ grade 2 antrum is uncommonly seen in the fasting state. Gastric ultrasound has been shown to be reliable and accurate to identify a 'full stomach'. 98116

Quantification of gastric antrum volume has been validated against endoscopically guided gastric suctioning ${ }^{110112}$ for a wide range of gastric volumes $(0-500 \mathrm{~mL})$, patient's body mass index (19-60) and ages (18-85 years). By placing the patient in the RLD position and measuring the cross-sectional area (CSA) of the gastric antrum at the level of the aorta, the following statistical model can be used to measure gastric volume. Gastric volume $(\mathrm{mL})=27.0+14.6 \times \mathrm{RLD}$ CSA $-1.28 \times$ age ${ }^{112}$ This statistically robust model has the benefit of only one covariate (age) and has been shown to have high intrarater and inter-rater reliability.

\section{Focused cardiac ultrasound}

FoCUS has been used in critical care for over 25 years, with the earliest example being the focus assessed transthoracic echocardiography (FATE) protocol. ${ }^{117}$ While echocardiography was, for decades, the exclusive domain of the cardiologist, those days have passed. Both the American Society of Echocardiography ${ }^{118}$ and the World Interactive Network Focused on Critical UltraSound $^{119}$ have published recommendations for the utility of
FoCUS at the bedside for specific clinical indications. With the availability of high-quality, hand-carried devices, as well as the expansion of training and expertise, the perioperative setting has been ushered into a new era of bedside ultrasound. Specifically, this evidence has been supplemented by several review articles describing the value of FoCUS in the broad practice of anesthesiology, ${ }^{120}{ }^{121}$ regional anesthesia and pain medicine. ${ }^{12} 122$ Although there are no controlled studies on the utilization of FoCUS in pain practice at this time, there are several scenarios where it could be clinically relevant.

FoCUS aids with patient assessment and clinical optimization, and it can help guide the management of procedural complications. Patients presenting urgently or emergently in the perioperative or periprocedural setting can have undiagnosed and potentially significant cardiac comorbidities. FoCUS can be used to screen high-risk patient populations for undiagnosed cardiac disease when formal echocardiography is not promptly available. ${ }^{118}$ One clinical example is the elderly patient presenting with a hip fracture. ${ }^{123}$ In a prospective study, hip fracture patients who received routine bedside cardiac ultrasound screening found that $31 \%$ of patients with no audible murmur had aortic stenosis (AS). ${ }^{124}$ Diagnosis of significant pathologies, such as AS, should alter anesthetic technique, monitoring and postoperative care. ${ }^{125}$ Additionally, bedside cardiac ultrasound on high-risk hip fracture patients did not delay entry into the operating room and may have improved short-term and long-term mortality. ${ }^{123}$

FoCUS can differentiate among intraoperative and periprocedural complications. In the setting of refractory hypotension following a procedure, FoCUS can differentiate among decreased preload seen with a high spinal, poor contractility related to LAST to the myocardium and other complications associated 

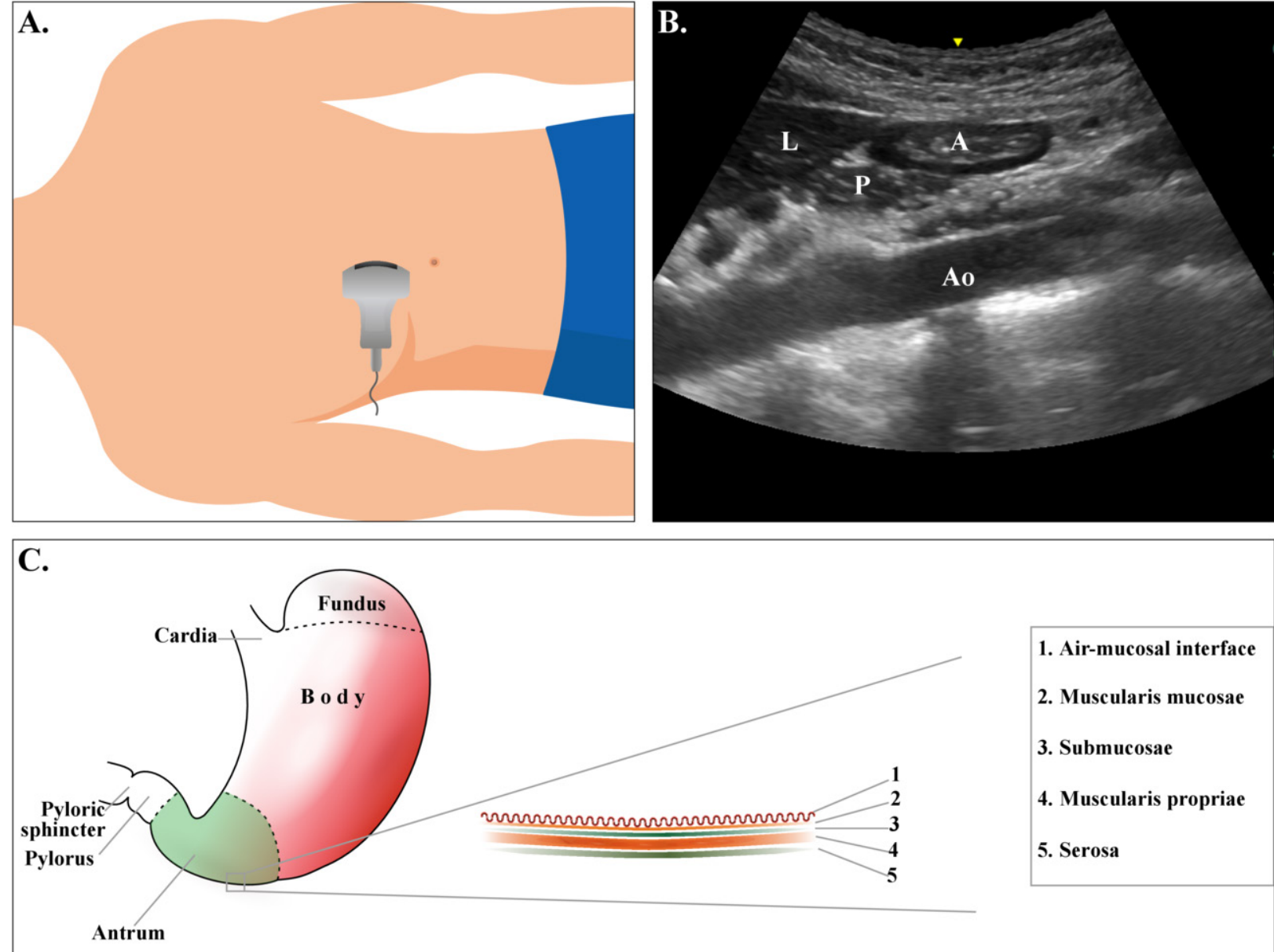

Figure 4 Gastric ultrasound probe placement and anatomy: (A) Probe placement for gastric ultrasound. (B) Basic sonoanatomy of the gastric ultrasound view at the level of the gastric antrum. (C) The five sonographic layers of the gastric wall. Note that with a low-frequency curvilinear transducer, the resolution is decreased and only the muscularis propria is evident. A, antrum; Ao, aorta; L, liver; P, pancreas.

with more complex cardiac pathologies (eg, myocardial infarction (MI), PE, undiagnosed pericardial effusion). ${ }^{126}$

FoCUS can also provide repeated evaluation of volume status and ventricular function, which is often not logistically feasible with formal echocardiography ${ }^{118}$ 127-129; additionally, FoCUS may prompt further testing or consultation. For example, although FoCUS has a low sensitivity for PE, ${ }^{130}{ }^{131}$ when there is a high level of clinical suspicion and visualization of a dilated right ventricle (RV), it might lead to a more urgent pursuit of definitive imaging like CT angiography (CTA). ${ }^{118}$ Similarly, while FoCUS should not be used to detect wall motion abnormalities, ${ }^{118} 119$ global left ventricle (LV) systolic dysfunction in a patient with ECG changes and other supportive symptoms may prompt more rapid cardiology consultation. Of note, negative findings with FoCUS in a clinical situation suspicious for the above pathologies should not discourage pursuing more conclusive investigations, such as ordering advanced imaging, for example, CTA or a complete transthoracic echocardiogram.

There are several clinical scenarios where the utilization of FoCUS can be considered for the chronic pain or interventional pain physician. Radiographic contrast is routinely used in interventional pain management to help detect an intravascular spread of the injectate. While adverse reactions are rare, they can be life-threatening. ${ }^{132}$ Therefore, pain physicians should be equipped with tools allowing timely management of an anaphylactic reaction or other complications. FoCUS can be potentially used to treat other rare complications of pain management procedures, including hypotension after sympathetic blocks or respiratory and cardiovascular collapse resulting from brainstem anesthesia after trigeminal blocks. ${ }^{133} 134$

\section{Cardiac arrest}

FoCUS is emerging as an important tool to assist with diagnosing and managing patients in cardiac arrest. ${ }^{135}$ FoCUS plays a role in the asystole and pulseless electrical activity (PEA) pathway in the advanced cardiac life support (ACLS) algorithm. FoCUS can help diagnose treatable pathologies such as hypovolemia, PE, LV failure and pericardial tamponade. FoCUS can also provide insight into prognosis and survivability based on the presence or absence of LV wall motion. ${ }^{126}$ The focused echocardiographic evaluation in life support protocol describes the optimal timing and FoCUS views to assist in diagnosis and management during ACLS while avoiding interference with other essential treatments. 126

\section{FoCUS views}

The fundamental FoCUS views are the parasternal long axis, parasternal short axis, apical four-chamber, subcostal fourchamber and the subcostal-inferior vena cava view. These views are sufficient to obtain the qualitative information needed to diagnose gross and potentially life-threatening pathology. This article will not detail view acquisition as there are multiple resources available in publications and online. ${ }^{12} 121{ }^{136}$ However, 


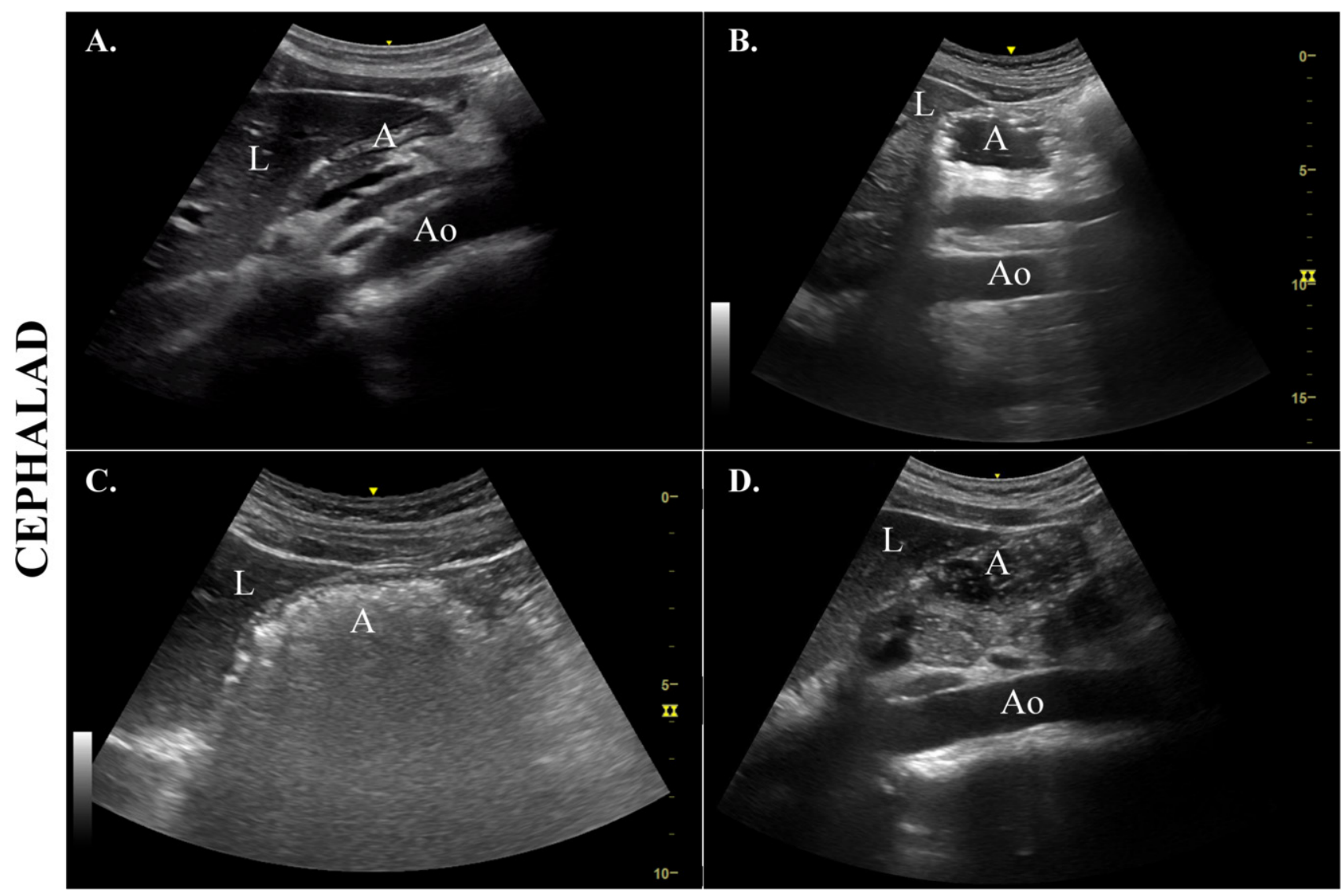

Figure 5 Gastric ultrasound sonoanatomy. (A) Sonographic appearance of the antrum in an empty stomach. (B) Appearance with clear fluid. (C) Appearance soon after a solid meal (early stage solid) with significant air content. (D) Appearance with mixed solid and fluid content (late stage solid). A, antrum; Ao, aorta; L, liver.

figure 6 highlights the basic views as initially described with the FATE protocol.

\section{Relevant pathology}

FoCUS can identify specific gross pathologies, and the following section will highlight some of the most pertinent pathologies for regional anesthesiologists and pain medicine physicians. Before implementing a new clinical skill, it is essential to understand the indications and subsequent steps required to practice this skill both safely and correctly. The I-AIM Framework is a standardized, step-by-step guide for clinicians learning a new POCUS skill to identify the appropriate clinical scenarios where it can be used. $^{48}{ }^{49}$ table 4 has a summary of each cardiac pathology using the I-AIM Framework.

\section{Local anesthetic systemic toxicity}

Although LAST is a rare complication for regional anesthesiologists and pain specialists, it is one of the most critical complications to assess and manage appropriately. LAST resulting from peripheral nerve blocks or neuraxial anesthesia can be devastating, causing severe ventricular arrhythmias, myocardial depression and cardiovascular collapse. There is a complex algorithm for the assessment and management of LAST, ${ }^{137}$ which is particularly relevant when LAST is detected immediately following local anesthetic injection. However, FoCUS can be essential with a delayed presentation of LAST, which can mimic other cardiac pathologies such as $\mathrm{MI}^{138}$ and cardiogenic shock. ${ }^{139}$ For example, in an otherwise healthy patient presenting with delayed hemodynamic instability following either neuraxial or peripheral nerve block, FoCUS can reveal new-onset myocardial dysfunction suggesting LAST.

\section{Aortic stenosis}

AS is often of concern for a patient presenting for urgent or emergent surgery (for example, hip fracture surgery) without a recent medical workup and an audible systolic murmur on auscultation. Hip fracture patients can benefit significantly from neuraxial technique depending on their comorbidities; however, a patient with severe AS is at risk for significant hemodynamic changes and potential complications following induction with neuraxial technique. Although the definitive diagnosis and grading of valvular AS require advanced technical skill and knowledge, FoCUS can be used to detect morphologic signs that suggest AS and prompt additional diagnostic imaging as well as changes in management. ${ }^{118119125140}$

\section{Hypovolemia}

Assessment for hypovolemia before a neuraxial blockade can add considerable value and help guide management. For example, a trauma patient presenting for emergent surgery with an occult bleed or a patient with end-stage renal disease who has recently undergone dialysis may have inadequate preload at baseline, resulting in significant hemodynamic changes and potential cardiac arrest following induction with neuraxial technique. Alternatively, hypovolemia may result from low vascular resistance and reduced afterload, as seen in pathologies such as sepsis or septic shock. 


\section{A. Probe Position}
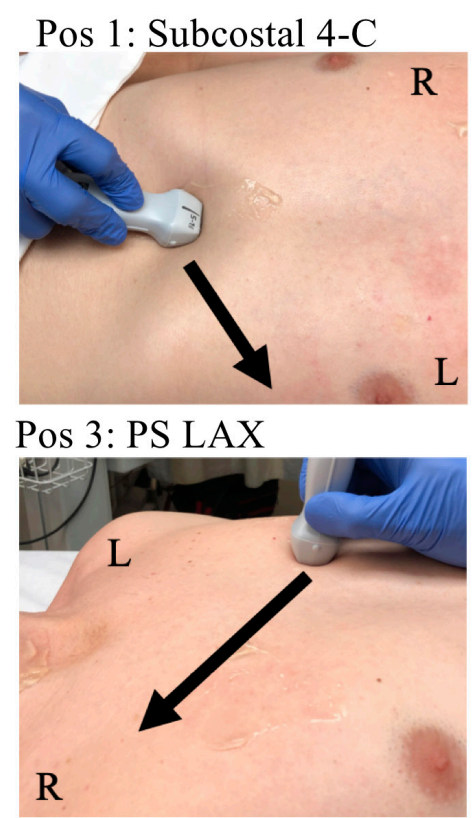

Position 1: Subcostal IVC

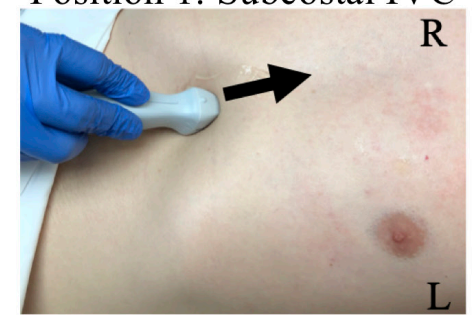

B. Modified FATE Card

regionmidtjylland ind

\section{Focus Assessed Transthoracic Echo (FATE)} Scan position 1-3 in the most favorable sequence

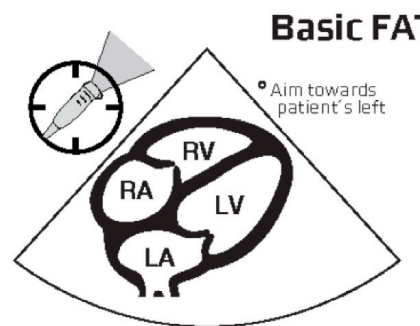

Pos 1: Subcostal 4-chamber

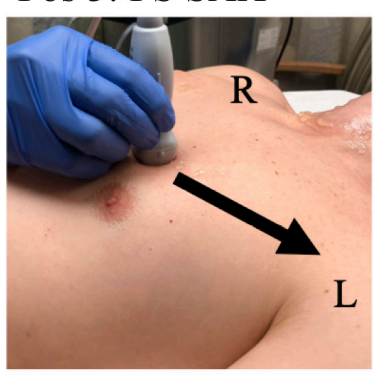

$\mathrm{L}$
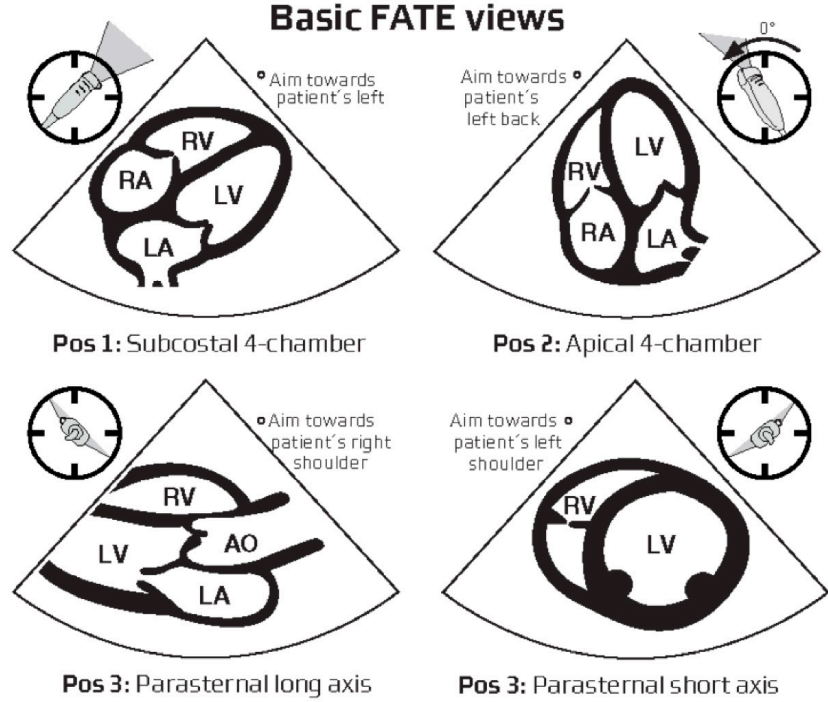

Pos 2: Apical 4-chamber

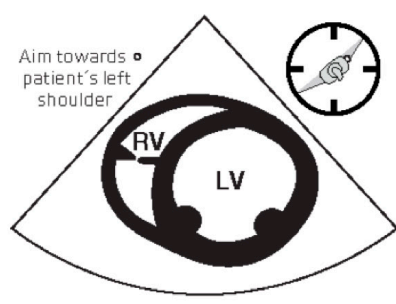

Pos 3: Parasternal short axis

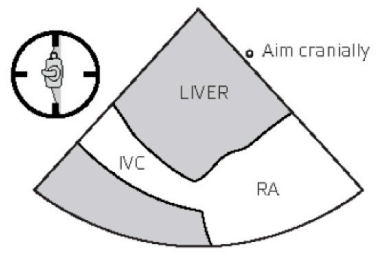

Pos 1: Subcostal Inferior Vena Cava

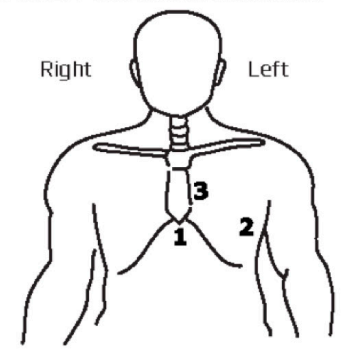

Figure 6 Probe placement for a focus cardiac ultrasound examination and modified basic FATE card to Include subcostal IVC view. (A) Probe placement for each FATE view with arrow demonstrating orientation marker direction. (B) Modified basic FATE card with IVC view. 4-C, four-chamber; Ao, ascending aorta; IVC, inferior vena cava view; L, left; LA, left atrium; LV, left ventricle; R, right; RA, right atrium; RV, right ventricle; PS LAX, parasternal long axis, PS SAX, parasternal short axis.

\section{Pulmonary embolism}

$\mathrm{PE}$ is a relatively common complication following orthopedic procedures $(\sim 1.7 \%)^{141}$ and is a significant cause of death following trauma surgery $(\sim 1.6 \%) .{ }^{142}$ Regional anesthesiologists routinely manage patients at risk for this complication, and it should be high on our differential diagnosis in the perioperative setting of hemodynamic instability and cardiovascular collapse. PE is a time-sensitive emergency that requires a rapid diagnosis to ensure adequate intervention and management. Although FoCUS has a low sensitivity for PE, ${ }^{130131}$ it has been shown to have high specificity in the setting of massive PE, particularly in patients without any known preexisting cardiovascular disease. ${ }^{143} 144$ Occlusion of the pulmonary vasculature, as seen with massive PE, causes an acute elevation in RV pressure resulting in RV dilation and failure. Although emboli are not always visible, multiple FoCUS findings significantly increase suspicion for PE and should direct further evaluation and/or management.

\section{Summary of clinical indications}

Based on the evidence presented, there are multiple clinical applications for POCUS; however, to provide additional examples to guide training, this group has provided several educational casebased clinical scenarios (online supplemental file 1 part I-CaseBased Clinical Scenarios) for review that can be implemented into a training program.

\section{Pediatric POCUS}

POCUS is becoming an essential tool for diagnostic and procedural purposes in pediatric acute care medicine, similar to its use in adult populations. ${ }^{145-149}$ Evidence documents the benefits of gastric, ${ }^{106150151}$ cardiac, $^{147} 148$ airway and lung ${ }^{152-156}$ ultrasound use in children. While there are many similarities between POCUS imaging for adults and children, the differing size, physiology, common injury patterns and distribution of comorbidities in children create aspects of POCUS that are unique to pediatric patients. ${ }^{15}$

When considering the various categories of the I-AIM model, most of the technical aspects of image acquisition are quite similar between children and adults. In general, smaller footprint, higher frequency transducers are used on children, and the depth and focus settings need to be adjusted to size. Otherwise, the views are acquired through the same windows with similar transducer orientations. Obtaining intraoperative images can be easier given that there is a shorter distance to the target structures. However, when infants and children are draped and positioned for surgery, their smaller size limits access to sonographic windows. Indications, interpretation and directed medical management differ due to the pathophysiologic characteristics of pediatric patients. The following sections summarize the distinct aspects of POCUS applications in children to supplement the didactic information already included in the previous sections. 
Table 4 FoCUS I-AIM framework for the regional anesthesiologist

\begin{tabular}{|c|c|c|c|c|}
\hline Pathology & Clinical indication & $\begin{array}{l}\text { Acquisition-optimal } \\
\text { FoCUS view(s) }\end{array}$ & Interpretation & Medical decision-making \\
\hline $\begin{array}{l}\text { Local anesthetic systemic } \\
\text { toxicity (LAST) }\end{array}$ & $\begin{array}{l}\text { Delayed onset of hemodynamic } \\
\text { instability following neuraxial or } \\
\text { PNB in a patient with no known } \\
\text { cardiac disease ( } \pm \text { arrhythmias) }\end{array}$ & $\begin{array}{l}\text { PSAX } \\
\text { Alternative Acceptable Views: } \\
\text { PLAX, A4C, SC4C }\end{array}$ & $\begin{array}{l}\text { PSAX } \\
\text { Sluggish, poorly contracting and } \\
\text { potential dyssynchronous LV }\end{array}$ & $\begin{array}{l}\text { Provide hemodynamic support } \\
\text { ( } \pm \text { inotropes or vasopressors) } \\
\text { Follow LAST guidelines, including the use } \\
\text { of lipid emulsion }\end{array}$ \\
\hline Aortic stenosis & $\begin{array}{l}\text { Urgent or emergent surgery } \\
\text { (eg, hip fracture surgery) with } \\
\text { inadequate medical workup and } \\
\text { an audible systolic murmur on } \\
\text { auscultation }\end{array}$ & $\begin{array}{l}\text { PLAX } \\
\text { PSAX at the level of } A V^{*}\end{array}$ & $\begin{array}{l}\text { PLAX and PSAX at level of AV* } \\
\downarrow A V \text { cusp mobility } \\
\uparrow A V \text { cusp thickness/calcification }\end{array}$ & $\begin{array}{l}\text { Urgent Case } \\
\text { Formal TTE and cardiology consultation } \\
\text { Emergent case } \\
\text { Adjust perioperative } \\
\text { management:+arterial line, } \\
\text { maintain hemodynamics with } \\
\text { vasopressors } \pm \text { inotropes throughout } \\
\text { induction, escalate postoperative care to } \\
\text { stepdown or ICU }\end{array}$ \\
\hline Hypovolemia & $\begin{array}{l}\text { Urgent or emergent surgery with } \\
\text { concern for hypovolemia (eg, } \\
\text { trauma patient or ESRD following } \\
\text { dialysis) }\end{array}$ & $\begin{array}{l}\text { SCIVC } \\
\text { PSAX }\end{array}$ & $\begin{array}{l}\text { SCIVC } \\
\text { IVC diameter }<1.5 \mathrm{~cm},>50 \% \\
\text { collapse with 'kissing wall sign' } \\
\text { PSAX } \\
\text { Hyperdynamic LV with underfilled } \\
\text { end-diastolic volume and } \\
\text { end-systolic 'touching' of the } \\
\text { myocardium } \\
\text { Note: Emptying depends on } \\
\text { afterload }\end{array}$ & $\begin{array}{l}\text { Fluid resuscitation prior to induction } \\
\text { Consider gradual induction with epidural } \\
\text { over a subarachnoid block }\end{array}$ \\
\hline Pulmonary embolism (PE) & $\begin{array}{l}\text { Suspected massive PE in high- } \\
\text { risk patient population (eg, } \\
\text { orthopedics or trauma patient) }\end{array}$ & $\begin{array}{l}\text { PSAX } \\
\text { A4C or SC4C }\end{array}$ & $\begin{array}{l}\text { PSAX } \\
\text { D-shaped septal shift, RV }>\text { LV } \\
\text { A4C or SC4C } \\
\text { RV and RA dilation }>\text { LV } \\
\text { Intraventricular septal bowing } \\
\text { toward LV during systole }\end{array}$ & $\begin{array}{l}\text { Cardiopulmonary resuscitation when } \\
\text { necessary } \\
\text { Initiate thrombolytic therapy and/or } \\
\text { embolectomy }\end{array}$ \\
\hline
\end{tabular}

${ }^{*}$ Advanced view not discussed in the recommendations.

A4C, apical four-chamber view; AV, aortic valve; ESRD, end-stage renal disease; FoCUS, focused cardiac ultrasound; I-AIM, Indication, Acquisition, Interpretation, and Medical Decision-Making Framework; ICU, intensive care unit; IVC, inferior vena cava view; LAST, local anesthetic systemic toxicity; LV, left ventricle; PLAX, parasternal long axis view; PNB, peripheral nerve block; PSAX, parasternal short axis view; RA, right atrium; RV, right ventricle; SC4C, subcostal four-chamber view; SCIVC, subcostal inferior vena cava view; TTE, transthoracic echocardiogram.

\section{Airway and LUS}

Infants and children have an increased risk of endobronchial intubation due to relatively short tracheal length. ${ }^{157}$ The ASA Closed Claims Project shows that bronchial intubations account for $4 \%$ of respiratory claims in children compared with $2 \%$ in adults. ${ }^{158}$ Point-of-care LUS correctly identifies endobronchial intubation in 95\%-100\% of children. ${ }^{153} 159160$ Diagnosis of an interstitial syndrome and pneumothorax is similar to adult data.

The proportionally large head, small mouth opening, bigger tongue and anterior position of the larynx in infants ${ }^{157}$ predisposes them to accidental esophageal intubation, which occurs in up to $21 \%$ of infants. ${ }^{161}$ Those patients with esophageal intubation have a $4 \%$ incidence of hypotension and a 3\% incidence of initiation of chest compression. ${ }^{161}$ Real-time tracheal ultrasound imaging using a high-frequency linear transducer placed transversely just above the sternal notch has a sensitivity and specificity of $98.5 \%-100 \%$ and $75 \%-100 \%$, respectively, for correctly diagnosing esophageal intubation. ${ }^{153} 159160$ Airway ultrasound has the potential to eliminate the need for test ventilation and speed the diagnosis of esophageal intubation.

\section{Abdominal ultrasound}

In response to hypovolemia, children have greater hemodynamic compensatory mechanisms to maintain blood pressure until $40 \%$ of the blood volume is lost, making a timely diagnosis of intra-abdominal bleeding via abdominal ultrasound advantageous. ${ }^{162-164}$ The smaller caliber of Foley catheters makes kinking and plugging common, and ultrasound of the bladder can help to differentiate causes of anuria.

\section{Gastric ultrasound}

The gastric volume is measured in pediatric patients in $\mathrm{mL} / \mathrm{kg}$ with a volume greater than $1.5 \mathrm{~mL} / \mathrm{kg}$ suggesting greater than baseline secretions. ${ }^{107}$ Children may not understand the importance of remaining NPO and may violate NPO guidelines when not directly observed. Comorbidities placing them at risk for delayed gastric emptying are short gut syndrome and pyloric stenosis. In infants presenting for a repair of pyloric stenosis, pre gastric and post gastric suctioning imaging can be used to direct the anesthetic induction technique. ${ }^{150}$ Gastric imaging is ideally performed pre induction, but children may not cooperate, making imaging difficult. Clinical applications of gastric ultrasound imaging in children are sparse. ${ }^{101150}$

\section{Focused cardiac ultrasound}

Given the prevalence of congenital heart disease in children, it is important to realize that bedside cardiac ultrasound has very limited applications in children with congenital heart disease and should not be used to delineate structural abnormalities. ${ }^{118} 147165$ Ultrasound imaging of the heart adds important information to the physical examination in children and is most commonly indicated in the setting of undifferentiated hypotension or tachycardia. ${ }^{147148166167}$ The use of cardiac ultrasound in the setting of pediatric cardiac arrest is distinct from adult 

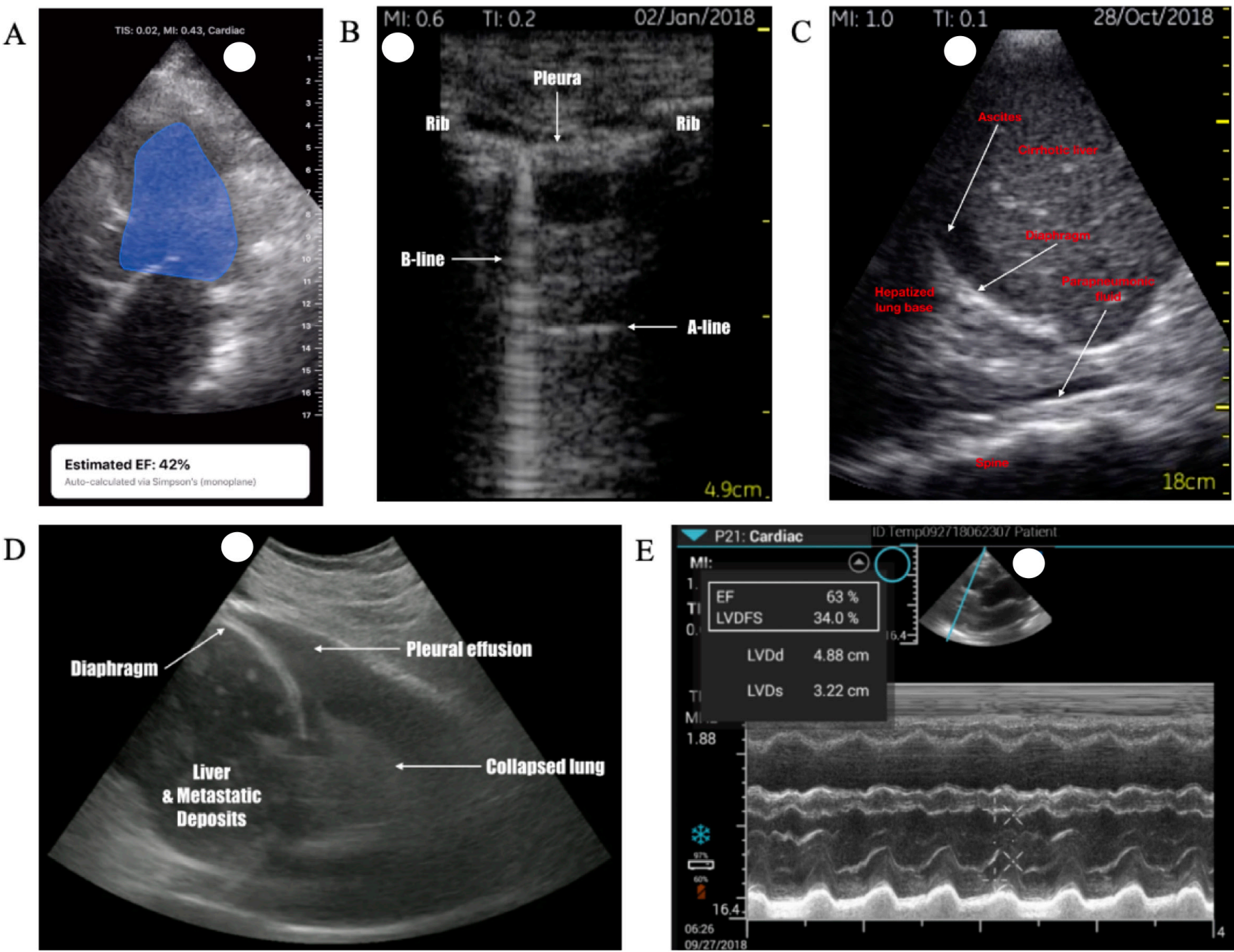

Figure 7 Various images obtained from a range of portable handheld devices. (A) Apical four-chamber view with auto-ejection fraction calculation. (B) LUS - upper zone view demonstrating anatomy and pathology. (C) Right lung base/right upper quadrant view showing cirrhotic liver, ascites and parapneumonic fluid. (D) Right upper quadrant abdominal scan with lung base view, demonstrating metastatic cancer in liver with pleural effusion. (E) Parasternal long axis of the heart, demonstrating calculation of fractional area change. EF, ejection fraction; LUS, lung ultrasound; LVDd; left ventricular end-diastolic diameter; LVDFS, left ventricular dimension fraction shortening; LVDs, left ventricular end-systolic diameter.

practice. While insufficient evidence exists to recommend for or against the routine use of FoCUS during pediatric cardiopulmonary resuscitation (CPR), the 2010 International Pediatric Basic and Advanced Life Support guidelines recommend that bedside cardiac echocardiography may be considered to identify potentially treatable causes of a cardiac arrest when appropriately skilled personnel are available, but the benefits must be carefully weighed against the known deleterious consequences of interrupting chest compression'. ${ }^{168}$ Ultrasound can be used to identify reversible causes of cardiac arrest, including cardiac tamponade, severe hypovolemia and pulmonary air embolus. ${ }^{126}$ 169-172 The diagnosis of cardiac standstill or PEA in children carries a different prognosis and algorithmic sequence compared with adults. Forty per cent of intraoperative cardiac arrests in children occur from a respiratory etiology, and restoration of oxygen delivery alone may lead to a return of spontaneous circulation. ${ }^{173}$ Extracorporeal membrane oxygenation for cardiac resuscitation (E-CPR) as a rescue strategy in children in cardiac arrest is not uncommon. ${ }^{174}$ There is evidence that E-CPR for pediatric patients with in-hospital arrest requiring $>10$ min of standard CPR is associated with improved survival and neurologic outcomes. There is also evidence that prolonged conventional CPR with ongoing use of epinephrine every 3-5 min (as recommended by pediatric advanced life support protocol) and resultant elevated systemic vascular resistance may have deleterious effects when implementing E-CPR by limiting extracorporeal membrane oxygenation pump flows. ${ }^{175}$ More rapid determination of myocardial standstill using cardiac ultrasound may facilitate more rapid progression to E-CPR with improvement in outcomes.

\section{Handheld devices in POCUS applications}

Increasingly portable ultrasound machines have led to an exponential uptake in utilization and discussion in the literature ${ }^{176}$ with some devices priced for individual purchase. ${ }^{177}{ }^{178}$ Traditional ultrasound machines have many barriers to use, including size, cost and difficulty to operate. Therefore, handheld devices can be an introductory device before becoming competent in advanced ultrasound applications. ${ }^{179}$ For example, the introduction of handheld ultrasound devices in medical schools aids with teaching functional anatomy and with implementing a clinical 
Box 1 Summary of advantages and disadvantages to handheld devices using an I-AIM Framework.

Advantages and disadvantages of handheld devices in POCUS applications:

Advantages

- Portability/convenience.

- Increased teaching and training opportunities due to portability/affordability.

- App-based software upgrades offering Al and other increased functionality.

- Ease of transport.

- Minimal boot-up time.

- Practical in confined spaces.

- Simple and less intimidating to beginners.

- Cloud-based storage available.

- WiFi image upload possible with DICOM linkage to existing institution systems.

- Potential to use existing electronic equipment (Androidbased/Apple-based devices).

Limitations and pitfalls

- Poorer image quality than higher-end systems:

- More prone to artifacts.

- Greater risk for image misinterpretation.

- Small screen size:

- Limits image acquisition.

- Prevents multiple functionalities on the screen.

- Increased phantom scanning (scanning without image capture/record).

- Limited compatibility with existing image storage system.

- Limited advanced functionality (ie, pulsed or continuous wave Doppler).

- Potential for overuse and misinterpretation of incidental findings.

- Misplacement, theft or loss of the device.

- Scanning time limited by battery life.

- Transducer selection is device dependent.

- Probes overheat.

- Cloud security/governance challenges

- Wireless devices prone to drop-out/'jumpy images'/limited image quality on probe movement.

- Sterility concerns.

$\mathrm{Al}$, artificial intelligence; DICOM, Digital Imaging and Communications in Medicine; POCUS, point-of-care ultrasound; WiFi, wireless fidelity.

POCUS curriculum. ${ }^{180}{ }^{181}$ Examples of basic images obtained from a selection of these devices are shown in figure 7.

Limitations in image quality, screen size, available memory and data encryption make handheld ultrasound devices better used to extend the physical examination rather than comprehensive diagnostics tools. Sterility is of utmost importance given the potential for frequent use and the ability to attach to personal devices.

Critics of POCUS often express concern that it deprioritizes patient contact during the physical examination; however, handheld ultrasound can bridge the gap between the clinician and diagnostic tests while facilitating communication with visual confirmation of findings in real time. ${ }^{182}$ The presentation of pathology can aid compliance and buy-in for additional testing/ imaging.
To offer a balanced approach to handheld devices, some of the advantages and disadvantages are shown in box 1 .

\section{SUMMARY}

POCUS is an essential skill for all regional anesthesiologists and pain physicians to help diagnose relevant complications related to routine practice and guide perioperative management. The growing evidence supporting the clinical utility of POCUS has been outlined to lay the foundation for part II of the recommendations. Part II will provide structured guidelines and strategies for the education of the trainee and postgraduate learner.

The expert panel acknowledges that clinical evidence supporting POCUS is evolving, and certain endpoints, such as improved survival during CPR, require additional validation. ${ }^{181} 182$ All interventions have risks or downsides, including potential false-negative findings, higher rates of interventions or delayed bedside management due to the performance of an examination. ${ }^{181} 182$ Because of these concerns, adequate training and future investigation into applications are essential for the adaptation of POCUS into the perioperative and periprocedural setting.

Regarding POCUS skills that fall outside of the traditional scope of regional anesthesiologists and pain specialists, such as the FAST examination, the expert panel emphasizes learning to perform these skills as well. As these skills are introduced into new clinical practice realms, there is great potential to illuminate novel clinical scenarios in the perioperative and periprocedural setting that can aid in patient assessment and management.

\section{Author affiliations}

Anesthesiology, Critical Care \& Pain Management, Hospital for Special Surgery, New York, New York, USA

${ }^{2}$ Anesthesiology, Weill Cornell Medical College, New York, New York, USA

${ }^{3}$ Anesthesiology, Duke University School of Medicine, Durham, North Carolina, USA

${ }^{4}$ Anesthesiology and Pain Management, Toronto Western Hospital, Toronto, Ontario,

Canada

${ }^{5}$ Anaesthesia, Guy's \& St Thomas' NHS Foundation Trust, London, UK

${ }^{6}$ Anesthesiology, University of Utah Health, Salt Lake City, Utah, USA

${ }^{7}$ Anesthesiology, Critical Care and Pain Medicine, Harvard Medical School, Boston,

Massachusetts, USA

${ }^{8}$ Anesthesiology, McMaster University, Hamilton, Ontario, Canada

${ }^{9}$ Anesthesiology, University of Michigan, Ann Arbor, Michigan, USA

${ }^{10}$ Anesthesiology, University of Texas Health Science Center at Houston, Houston,

Texas, USA

${ }^{11}$ Anesthesiology, Stanford Hospital and Clinics, Stanford, California, USA

${ }^{12}$ Anesthesiology, University of Virginia Health System, Charlottesville, Virginia, USA

${ }^{13}$ Anaesthesia, Belfast Health and Social Care Trust, Belfast, UK

${ }^{14}$ Intensive Care and Anaesthesia, Northampton General Hospital, Northampton, UK

${ }^{15}$ Anesthesiology, UAB, Birmingham, Alabama, USA

${ }^{16}$ Anesthesiology, Loma Linda University Medical Center, Loma Linda, California, USA

${ }^{7}$ Anesthesiology, Clement Zablocki VA Medical Center/Medical College of

Wisconsin, Milwaukee, Wisconsin, USA

${ }^{18}$ Anesthesiology, Northwestern University Feinberg School of Medicine, Chicago,

Illinois, USA

${ }^{19}$ Center for Pain Medicine, Western Reserve Hospital, Cuyahoga Falls, Ohio, USA

Twitter Stephen C Haskins @shaskinsMD, Anahi Perlas @PerlasAnahi, Kariem ElBoghdadly @elboghdadly, Jan Boublik @janboublik MDPhD, Hariharan Shankar @ hariharan_shank and Samer Narouze @NarouzeMD

Acknowledgements The authors would like to thank the American Society of Regional Anesthesia and Pain Medicine's (ASRA) Guidelines Committee and Board of Directors for their feedback and support in the design of the recommendations. The authors would also like to thank Professor Erik Sloth, MD, PhD, for his ongoing inspiration as well as permission to present an adaptation of the focus assessed transthoracic echo card.

Contributors All authors are responsible for the content and have read and approved the manuscript for submission to the American Society of Regional Anesthesia and Pain Medicine. 
Funding Anahi Perlas, MD receives funding from a Merit Award, Department of Anesthesiology and Pain Medicine, University of Toronto.

Competing interests None declared.

Patient consent for publication Not required.

Provenance and peer review Not commissioned; externally peer reviewed.

Data availability statement There are no data in this work.

ORCID iDs

Stephen C Haskins http://orcid.org/0000-0001-8012-7594

Anahi Perlas http://orcid.org/0000-0002-8190-8314

Kariem El-Boghdadly http://orcid.org/0000-0002-9912-717X

William Clark Manson http://orcid.org/0000-0002-6308-9036

Davinder Ramsingh http://orcid.org/0000-0002-8670-7300

Samer Narouze http://orcid.org/0000-0003-1849-1402

\section{REFERENCES}

1 Neal JM, Brull R, Chan VWS. The ASRA evidence-based medicine assessment of ultrasound-guided regional anesthesia and pain medicine: Executive summary. In: Regional Anesthesia and Pain Medicine. Vol 35. Reg Anesth Pain Med 2010.

2 Sites BD, Chan VW, Neal JM, et al. The American Society of regional anesthesia and pain medicine and the European Society of regional anaesthesia and pain therapy joint Committee recommendations for education and training in ultrasound-guided regional anesthesia. Reg Anesth Pain Med 2010;35:S74-80.

3 Narouze SN, Provenzano D, Peng P, et al. The American Society of regional anesthesia and pain medicine, the European Society of regional anaesthesia and pain therapy, and the Asian Australasian Federation of pain societies joint Committee recommendations for education and training in ultrasound-guided interventional pain procedures. Reg Anesth Pain Med 2012;37:657-64.

4 American Medical Association. House of Delegates policy H-230. 960. Privileging for ultrasound imaging. 2001. Updated 05/ 2016. [Epub ahead of print: Accessed].

5 Emergency ultrasound guidelines. Ann Emerg Med 2009;53:550-70.

6 Fagley RE, Haney MF, Beraud A-S, et al. Critical care basic ultrasound learning goals for American anesthesiology critical care trainees: recommendations from an expert group. Anesth Analg 2015;120:1041-53.

7 Frankel HL, Kirkpatrick AW, Elbarbary M, et al. Guidelines for the appropriate use of bedside general and cardiac ultrasonography in the evaluation of critically ill Patients—Part I. Crit Care Med 2015;43:2479-502.

8 American Society of Anesthesiologists (ASA) Ad Hoc Committee on Point-of-Care Ultrasound. Committee work product on diagnostic point-of-care ultrasound, 2019. Available: https://www.asahq.org/standards-and-guidelines/resources-from-asacommittees\#POM [Accessed 9 Oct 2020].

9 Haskins SC, Boublik J, Wu CL. Point-Of-Care ultrasound for the regional anesthesiologist and pain specialist: a series introduction. Reg Anesth Pain Med 2017:42:281-2.

10 Haskins SC, Tsui BC, Nejim JA, et al. Lung ultrasound for the regional anesthesiologist and acute pain specialist. Reg Anesth Pain Med 2017;42:289-98.

11 Haskins SC, Tanaka CY, Boublik J, et al. Focused cardiac ultrasound for the regional anesthesiologist and pain specialist. Reg Anesth Pain Med 2017;42:632-44.

12 Haskins SC, Kruisselbrink R, Boublik J, et al. Gastric ultrasound for the regional anesthesiologist and pain specialist. Reg Anesth Pain Med 2018;42:1.

13 Manson WC, Kirksey M, Boublik J, et al. Focused assessment with sonography in trauma (fast) for the regional anesthesiologist and pain specialist. Reg Anesth Pain Med 2019:44:540-8.

14 Kars MS, Gomez Morad A, Haskins SC, et al. Point-Of-Care ultrasound for the pediatric regional anesthesiologist and pain specialist: a technique review. Reg Anesth Pain Med 2020;45:rapm-2020-101341.

15 Patel SG, Benninger B, Mirjalili SA. Integrating ultrasound into modern medical curricula. Clinical Anatomy 2017:30:452-60.

16 Bertrand PB, Levine RA, Isselbacher EM, et al. Fact or artifact in two-dimensional echocardiography: avoiding misdiagnosis and missed diagnosis. J Am Soc Echocardiogr 2016;29:381-91

17 Quien MM, Saric M. Ultrasound imaging artifacts: how to recognize them and how to avoid them. Echocardiography 2018;35:1388-401.

18 Brenner K, Ergun A, Firouzi K, et al. Advances in capacitive micromachined ultrasonic transducers. Micromachines 2019:10:152.

19 Prabhu SJ, Kanal K, Bhargava P, et al. Ultrasound artifacts: classification, applied physics with illustrations, and imaging appearances. Ultrasound Q 2014;30:145-57.

20 Khuri-Yakub BT, Oralkan O. Capacitive micromachined ultrasonic transducers for medical imaging and therapy. J Micromech Microeng 2011;21:054004.

21 Lee W, Roh Y. Ultrasonic transducers for medical diagnostic imaging. Biomed Eng Lett 2017;7:91-7.

22 Meineri M, Bryson GL, Arellano R, et al. Core point-of-care ultrasound curriculum: what does every anesthesiologist need to know? Can J Anesth/J Can Anesth 2018;65:417-26
23 Ma IWY, Arishenkoff S, Wiseman J, et al. Internal medicine point-of-care ultrasound curriculum: consensus recommendations from the Canadian internal medicine ultrasound (CIMUS) group. J Gen Intern Med 2017;32:1052-7.

24 Ultrasound guidelines: emergency, point-of-care and clinical ultrasound guidelines in medicine. Ann Emerg Med 2017;69:e27-54.

25 Arntfield RT, Millington SJ, Ainsworth CD. Canadian recommendations for critical care ultrasound training and competency for the Canadian critical care Society. Canadian Respiratory Journal 2014:21:341-5.

26 Volpicelli G, Elbarbary M, Blaivas M. International liaison Committee on lung ultrasound (ILC-LUS) for international consensus conference on lung ultrasound (ICC-LUS). International evidence based recommendations for point-of-care lung ultrasound. Intensive Care Med 2012;38:557-91.

27 Moore CL. Does ultrasound improve clinical outcomes? prove It*. Crit Care Med 2015:43:2682-3

28 Atkinson PR, Milne J, Diegelmann L, et al. Does point-of-care ultrasonography improve clinical outcomes in emergency department patients with undifferentiated hypotension? an international randomized controlled trial from the SHoC-ED Investigators. Ann Emerg Med 2018;72:478-89.

29 Alessandri F, Antenucci G, Piervincenzi E. Ultrasound as a new tool in the assessment of airway difficulties: an observational study. European Journal of Anaesthesiology 2019;36:509-15

30 Kristensen MS, Teoh WH, Rudolph SS, et al. A randomised cross-over comparison of the transverse and longitudinal techniques for ultrasound-guided identification of the cricothyroid membrane in morbidly obese subjects. Anaesthesia 2016;71:675-83.

31 Kristensen MS, Teoh WH, Rudolph SS. Ultrasonographic identification of the cricothyroid membrane: best evidence, techniques, and clinical impact. Br J Anaesth 2016:117:i39-48.

32 Dixit A, Ramaswamy KK, Perera S, et al. Impact of change in head and neck position on ultrasound localisation of the cricothyroid membrane: an observational study. Anaesthesia 2019;74:29-32.

33 Zieleskiewicz L, Bouvet L, Einav S, et al. Diagnostic point-of-care ultrasound: applications in obstetric anaesthetic management. Anaesthesia 2018;73:1265-79.

34 Desai D, You-Ten KE, Arzola C, et al. Improved cricothyrotomy outcomes in human cadavers using ultrasound-guided compared to conventional digital palpation. J Clin Anesth 2014;26:166-7.

35 Siddiqui N, Arzola C, Friedman Z, et al. Ultrasound improves cricothyrotomy success in cadavers with poorly defined neck anatomy. Anesthesiology 2015:123:1033-41

36 Lee $\mathrm{D}, \mathrm{Czech} A \mathrm{~J}$, Elriedy M, et al. A multicentre prospective cohort study of the accuracy of conventional landmark technique for Cricoid localisation using ultrasound scanning. Anaesthesia 2018;73:1229-34.

37 Williams P, Umranikar A. A comparative prospective cohort study comparing physical exam to ultrasound for identifying the Cricoid cartilage. J Perioper Pract 2017;27:186-91.

38 Das SK, Choupoo NS, Haldar R. Vérification Du positionnement Du tube endotrachéa PAR échographie transtrachéale: revue systématique de la littérature et métaanalyse. Canadian Journal of Anesthesia 2015;62:413-23.

39 Ramsingh D, Frank E, Haughton R, et al. Auscultation versus Point-of-care Ultrasound to Determine Endotracheal versus Bronchial Intubation. Anesthesiology 2016;124:1012-20.

40 Adi 0, Kok MS, Abdull Wahab SF. Focused airway ultrasound: an armamentarium in future airway management. J Emerg Crit Care Med 2019;3:31.

41 Vigneau C, Baudel J-L, Guidet B, et al. Sonography as an alternative to radiography for nasogastric feeding tube location. Intensive Care Med 2005;31:1570-2.

$42 \mathrm{Kim} \mathrm{H}$, So B, Jeong W, et al. The effectiveness of ultrasonography in verifying the placement of a nasogastric tube in patients with low consciousness at an emergency center. Scand I Trauma Resusc Emerg Med 2012;20:38.

43 Brun P-M, Chenaitia H, Bessereau J, et al. Contrôle échographique de la position de la Sonde nasogastrique en préhospitalier. Annales Françaises d'Anesthésie et de Réanimation 2012:31:416-20.

44 Ahmad I, El-Boghdadly K, Bhagrath R, et al. Difficult airway Society guidelines for awake tracheal intubation (ATI) in adults. Anaesthesia 2020;75:509-28.

$45 \mathrm{~K}$ Ukey A, Matam S. Ultrasound guided stellate ganglion block for ventricular tachycardia storm. Indian Journal of Clinical Anaesthesia 2019;6:620-2.

46 Narouze S. Ultrasound-Guided stellate ganglion block: safety and efficacy. Curr Pain Headache Rep 2014;18:424.

47 Bahner DP, Hughes D, Royall NA. I-AIM: a novel model for teaching and performing focused sonography. J Ultrasound Med 2012;31:295-300.

48 Laursen CB, Nielsen K, Riishede M, et al. A framework for implementation, education, research and clinical use of ultrasound in emergency departments by the Danish Society for emergency medicine. Scand I Trauma Resusc Emerg Med 2014;22:25.

49 Goffi A, Kruisselbrink R, Volpicelli G. The sound of air: point-of-care lung ultrasound in perioperative medicine. Can J Anesth/J Can Anesth 2018:65:399-416.

50 CJ Y, Yang PC, HD W. Ultrasound study in unilateral hemithorax opacification: image comparison with computed tomography. American Review of Respiratory Disease 1993;147:430-4. 
51 Ding W, Shen Y, Yang J, et al. Diagnosis of pneumothorax by radiography and ultrasonography. Chest 2011;140:859-66.

52 Ye X, Xiao H, Chen B, et al. Accuracy of lung ultrasonography versus chest radiography for the diagnosis of adult community-acquired pneumonia: review of the literature and meta-analysis. PLOS One 2015;10:e0130066.

53 Al Deeb M, Barbic S, Featherstone R, et al. Point-Of-Care ultrasonography for the diagnosis of acute cardiogenic pulmonary edema in patients presenting with acute dyspnea: a systematic review and meta-analysis. Academic Emergency Medicine 2014;21:843-52.

54 Alrajab S, Youssef AM, Akkus NI, et al. Pleural ultrasonography versus chest radiography for the diagnosis of pneumothorax: review of the literature and metaanalysis. Crit Care 2013;17:R208

55 Yousefifard M, Baikpour M, Ghelichkhani P. Screening performance characteristic of ultrasonography and radiography in detection of pleural effusion; a meta-analysis. Emergency 2016:4:1-10.

56 Testa A, Soldati G, Copetti R, et al. Early recognition of the 2009 pandemic influenza A (H1N1) pneumonia by chest ultrasound. Critical Care 2012;16:R30.

57 Tardella M, Gutierrez M, Salaffi F, et al. Ultrasound in the assessment of pulmonary fibrosis in connective tissue disorders: correlation with high-resolution computed tomography. J Rheumatol 2012;39:1641-7.

58 Urmey WF, McDonald M. Hemidiaphragmatic paresis during interscalene brachial plexus block: effects on pulmonary functin and chest wall mechanics. Anesthesia and Analgesia 1992;74:352-257.

59 Matamis D, Soilemezi E, Tsagourias M, et al. Sonographic evaluation of the diaphragm in critically ill patients. technique and clinical applications. Intensive Care Med 2013;39:801-10.

60 Zambon M, Cabrini L, Beccaria P, et al. Ultrasound in critically ill patients: focus on diaphragm. Intensive Care Med 2013;39:986.

61 Lichtenstein D. Lung ultrasound in acute respiratory failure an introduction to the BLUE-protocol. Minerva Anestesiol 2009;75:313-7.

62 Lichtenstein D. Fluid administration limited by lung sonography: the place of lung ultrasound in assessment of acute circulatory failure (the FALLS-protocol). Expert Rev Respir Med 2012;6:155-62.

63 Lichtenstein DA. BLUE-protocol and FALLS-protocol: two applications of lung ultrasound in the critically ill. Chest 2015;147:1659-70.

64 Montoro E, Ferré F, Yonis H, et al. Pneumothorax as a complication of ultrasoundguided interscalene block for shoulder surgery. Eur J Anaesthesiol 2013:30:90-1.

65 Abell DJ, Barrington MJ. Pneumothorax after ultrasound-guided supraclavicular block: presenting features, risk, and related training. Regional Anesthesia and Pain Medicine 2014:39:164-7.

66 Ueshima H. Pneumothorax after the erector spinae plane block. J Clin Anesth 2018;48:12.

67 Park JS, Kim YH, Jeong SA, et al. Ultrasound-Guided aspiration of the iatrogenic pneumothorax caused by paravertebral block -A case Report-. Korean I Pain 2012;25:33-7.

68 Morjaria JB, Lakshminarayana UB. Liu Shiu Cheong P, Kastelik JA. pneumothorax: a tale of pain or spontaneity. Therapeutic Advances in Chronic Disease 2014:5:269-73.

69 Holzer A, Kapral S, Hellwagner K, et al. Severe pneumothorax after intercostal nerve blockade: a case report. Acta Anaesthesiol Scand 1998:42:1124-6.

70 Fitzgibbon DR, Posner KL, Domino KB. Chronic pain management: American Society of Anesthesiologists closed claims project. Anesthesiology 2004; 100:98-105

71 Radpay B, Parsa T, Dabir S. Acute respiratory failure as a rare complication of celiac plexus block in a patient with adenocarcinoma of the ampulla of Vater. Tanaffos 2012;11:54-7 http://www.ncbi.nlm.nih.gov/pubmed/25191416

72 Bergmann L, Martini S, Kesselmeier M, et al. Phrenic nerve block caused by interscalene brachial plexus block: breathing effects of different sites of injection. BMC Anesthesiol 2015;16:45.

73 Singh S, Katyal S, Kumar A, et al. Massive hemothorax: a rare complication after supraclavicular brachial plexus block. Anesthesia: Essays and Researches 2014;8:410

74 Hong J-M, Kim D, Kim S-W. Fatal hemothorax after thoracic epidural procedure for postoperative pain control in elderly esophageal cancer patient. J Thorac Dis 2018:10:E643-5.

75 Souron V, Reiland Y, Delaunay L. Pleural effusion and chest pain after continuous interscalene brachial plexus block. Reg Anesth Pain Med 2003:28:535-8.

76 Yang $\mathrm{CW}$, Jung SM, Cho CK, et al. Pleural effusion and atelectasis during continuous interscalene brachial plexus block -A case report-. Korean J Anesthesiol 2010;58:95-8.

77 Lichtenstein DA, Mezière GA. Relevance of lung ultrasound in the diagnosis of acute respiratory Failure*: the blue protocol. Chest 2008;134:117-25.

78 Beaulieu Y, Marik PE. Bedside ultrasonography in the ICU. Chest 2005;128:1766-81.

79 Ford JW, Heiberg J, Brennan AP, et al. A pilot assessment of 3 point-of-care strategies for diagnosis of perioperative lung pathology. Anesth Analg 2017;124:734-42.

80 Holm JH, Frederiksen CA, Juhl-Olsen P, et al. Perioperative use of focus assessed transthoracic echocardiography (fate). Anesthesia \& Analgesia 2012;115:1029-32.
81 OJ M, Mateer JR, Ogata M. Prospective analysis of a rapid trauma ultrasound examination performed by emergency physicians. Journal of Trauma - Injury, Infection and Critical Care 1995;38:879-85.

82 Haskins SC, Desai NA, Fields KG, et al. Diagnosis of intraabdominal fluid extravasation after hip arthroscopy with point-of-care ultrasonography can identify patients at an increased risk for postoperative pain. Anesthesia \& Analgesia 2017; 124:791-9

83 Rozycki GS, Ballard RB, Feliciano DV, v FD, et al. Surgeon-Performed ultrasound for the assessment of truncal injuries: lessons learned from 1540 patients. Ann Surg 1998:228:557-67.

84 Oba T, Hasegawa J, Sekizawa A. Postpartum ultrasound: postpartum assessment using ultrasonography. J Matern Fetal Neonatal Med 2017:30:1726-9.

85 Postpartum hemorrhage emergency management using focused assessment with sonography for obstetrics (Faso): a case report - POCUS Journal. Available: https:// pocusjournal.com/article/2019-04-02p17-19/ [Accessed December 7, 2020].

86 Branney SW, Wolfe RE, Moore EE, et al. Quantitative sensitivity of ultrasound in detecting free intraperitoneal fluid. J Trauma 1995:39:375-80.

87 Warner NS, Moeschler SM, Warner MA. Bleeding complications in patients undergoing celiac plexus block. Regional Anesthesia and Pain Medicine 2016;41:488-93

88 Van de Putte $\mathrm{P}$, Perlas A. Ultrasound assessment of gastric content and volume. $\mathrm{Br} J$ Anaesth 2014:113:12-22.

89 Carr CM, Plastaras CT, Pingree MJ, et al. Immediate adverse events in interventional pain procedures: a multi-institutional study. Pain Med 2016;17:2155-61.

90 Abrecht CR, Saba R, Greenberg P, et al. A contemporary medicolegal analysis of outpatient interventional pain procedures. Anesthesia \& Analgesia 2019;129:255-62.

91 Practice guidelines for preoperative fasting and the use of pharmacologic agents to reduce the risk of pulmonary aspiration: application to healthy patients undergoing elective procedures. Anesthesiology 2017;126:376-93.

92 Thapa N, Kappus M, Hurt R, et al. Implications of the opioid epidemic for the clinical gastroenterology practice. Curr Gastroenterol Rep 2019;21.

93 Camilleri M, Chedid V, Ford AC, et al. Gastroparesis. Nat Rev Dis Primers 2018:4:41.

94 Gola W, Domagała M, Cugowski A. Ultrasound assessment of gastric emptying and the risk of aspiration of gastric contents in the perioperative period. Anaesthesiology Intensive Therapy 2018;50:297-302.

95 Perlas A, Arzola C, Van de Putte P. Point-Of-Care gastric ultrasound and aspiration risk assessment: a narrative review. Can J Anaesth 2018;65:437-48.

96 Perlas A, Van de Putte P, Van Houwe P, et al. I-AIM framework for point-of-care gastric ultrasound. Br J Anaesth 2016;116:7-11.

97 Kruisselbrink R, Arzola C, Endersby R, et al. Intra- and interrater reliability of ultrasound assessment of gastric volume. Anesthesiology 2014;121:46-51.

98 Alakkad H, Kruisselbrink R, Chin KJ, et al. Point-Of-Care ultrasound defines gastric content and changes the anesthetic management of elective surgical patients who have not followed fasting instructions: a prospective case series. Canadian Journal of Anesthesia/Journal canadien d'anesthésie 2015;62:1188-95.

99 van de Putte P, van Hoonacker J, Perlas A. Gastric ultrasound to guide anesthetic management in elective surgical patients non-compliant with fasting instructions: a retrospective cohort study. Minerva Anestesiol 2018:84:787-95.

100 Boretsky KR, Perlas A. Gastric ultrasound imaging to direct perioperative care in pediatric patients: a report of 2 cases. A A Practice 2019;13:443-5.

101 Gagey A-C, de Queiroz Siqueira M, Monard C, et al. The effect of pre-operative gastric ultrasound examination on the choice of general anaesthetic induction technique for non-elective paediatric surgery. A prospective cohort study. Anaesthesia 2018;73:304-12.

102 Perlas A, Chan VWS, Lupu CM, et al. Ultrasound assessment of gastric content and volume. Anesthesiology 2009;111:82-9.

103 Perlas A, Davis L, Khan M. Gastric sonography in the fasted surgical patient: a prospective descriptive study. Anesthesia and Analgesia 2011;113:93-7.

104 Bouvet L, Desgranges F-P, Aubergy C, et al. Prevalence and factors predictive of full stomach in elective and emergency surgical patients: a prospective cohort study. $\mathrm{Br} J$ Anaesth 2017:118:372-9.

105 Spencer AO, Walker AM, Yeung AK, et al. Ultrasound assessment of gastric volume in the fasted pediatric patient undergoing upper gastrointestinal endoscopy: development of a predictive model using endoscopically suctioned volumes. Paediatr Anaesth 2015;25:301-8.

106 Bouvet L, Bellier N, Gagey-Riegel A-C, et al. Ultrasound assessment of the prevalence of increased gastric contents and volume in elective pediatric patients: a prospective cohort study. Pediatric Anesthesia 2018:28:906-13.

107 Arzola C, Perlas A, Siddiqui NT, et al. Gastric ultrasound in the third trimester of pregnancy: a randomised controlled trial to develop a predictive model of volume assessment. Anaesthesia 2018;73:295-303.

108 Desgranges F-P, Simonin M, Barnoud S, et al. Prevalence and prediction of higher estimated gastric content in parturients at full cervical dilatation: a prospective cohort study. Acta Anaesthesiol Scand 2019;63:27-33.

109 Kruisselbrink R, Arzola C, Jackson T, et al. Ultrasound assessment of gastric volume in severely obese individuals: a validation study. Br J Anaesth 2017;118:77-82. 
110 Van de Putte P, Perlas A. Gastric sonography in the severely obese surgical patient: a feasibility study. Anesth Analg 2014;119:1105-10.

111 Perlas A, Mitsakakis N, Liu L, et al. Validation of a mathematical model for ultrasound assessment of gastric volume by gastroscopic examination. Anesthesia \& Analgesia 2013;116:357-63.

112 Jay L, Zieleskiewicz L, Desgranges F-P, et al. Determination of a cut-off value of antral area measured in the supine position for the fast diagnosis of an empty stomach in the parturient. Eur J Anaesthesiol 2017:34:150-7.

113 Cubillos J, Tse C, Chan VWS, et al. Bedside ultrasound assessment of gastric content: an observational study. Can J Anesth/J Can Anesth 2012;59:416-23.

114 Bouvet L, Barnoud S, Desgranges F.-P., et al. Effect of body position on qualitative and quantitative ultrasound assessment of gastric fluid contents. Anaesthesia 2019;74:862-7.

115 Kruisselbrink R, Gharapetian A, Chaparro LE, et al. Diagnostic accuracy of point-ofcare gastric ultrasound. Anesth Analg 2019:128:89-95.

116 Jensen MB, Sloth E, Larsen KM, et al. Transthoracic echocardiography for cardiopulmonary monitoring in intensive care. Eur J Anaesthesiol 2004;21:700-7.

117 Spencer KT, Kimura BJ, Korcarz CE, et al. Focused cardiac ultrasound: recommendations from the American Society of echocardiography. J Am Soc Echocardiogr 2013;26:567-81.

$118 \mathrm{Via} \mathrm{G}$, Hussain A, Wells M, et al. International evidence-based recommendations for focused cardiac ultrasound. J Am Soc Echocardiogr 2014;27:683.e1-33.

119 Zimmerman JM, Coker BJ. The nuts and bolts of performing focused cardiovascular ultrasound (focus). Anesthesia \& Analgesia 2017;124:753-60.

120 Coker BJ, Zimmerman JM. Why Anesthesiologists must incorporate focused cardiac ultrasound into daily practice. Anesthesia \& Analgesia 2017;124:761-5.

121 Ramsingh D, Bronshteyn YS, Haskins S. Perioperative point-of-care ultrasound: from concept to application. Anesthesiology. Published online 2020.

122 Canty DJ, Royse CF, Kilpatrick D, et al. The impact on cardiac diagnosis and mortality of focused transthoracic echocardiography in hip fracture surgery patients with increased risk of cardiac disease: a retrospective cohort study. Anaesthesia 2012;67:1202-9.

123 Loxdale SJ, Sneyd JR, Donovan A, et al. The role of routine pre-operative bedside echocardiography in detecting aortic stenosis in patients with a hip fracture* Anaesthesia 2012;67:51-4.

124 Nishimura RA, Otto CM, Bonow RO. 2014 AHA/ACC guideline for the management of patients with valvular heart disease: Executive summary :A report of the american college of cardiology/american heart association task force on practice guidelines. Circulation 2014;129:2440-92

125 Breitkreutz R, Price S, Steiger HV, et al. Focused echocardiographic evaluation in life support and peri-resuscitation of emergency patients: a prospective trial. Resuscitation 2010:81:1527-33.

126 Canty DJ, Royse CF. Audit of anaesthetist-performed echocardiography on perioperative management decisions for non-cardiac surgery. Br $J$ Anaesth 2009;103:352-8

127 Cowie B. Three years' experience of focused cardiovascular ultrasound in the perioperative period. Anaesthesia 2011;66:268-73.

128 Shillcutt SK, Markin NW, Montzingo CR, et al. Use of rapid "rescue" perioperative echocardiography to improve outcomes after hemodynamic instability in noncardiac surgical patients. J Cardiothorac Vasc Anesth 2012;26:362-70.

129 Jackson RE, Rudoni RR, Hauser AM, et al. Prospective evaluation of twodimensional transthoracic echocardiography in emergency department patients with suspected pulmonary embolism. Academic Emergency Medicine 2000;7:994-8

130 Miniati M, Monti S, Pratali L, et al. Value of transthoracic echocardiography in the diagnosis of pulmonary embolism: results of a prospective study in unselected patients. Am J Med 2001;110:528-35.

131 Lee SH, Park JW, Hwang BM. Anaphylactic shock following nonionic contrast medium during caudal epidural injection. Korean J Pain 2015;28:280-3.

132 Saito T, Den S, Cheema SPS, et al. A single-injection, multi-segmental paravertebral block - extension of somatosensory and sympathetic block in volunteers (Note). Acta Anaesthesiol Scand 2001;45:30-3.

133 Robert SW, Feuerman T, Brown J. Neurogenic pulmonary edema after trigeminal nerve blockade. Chest 1989:96:436-8.

134 Link MS, Berkow LC, Kudenchuk PJ. Part 7: adult advanced cardiovascular life support: 2015 American heart association guidelines update for cardiopulmonary resuscitation and emergency cardiovascular care. Circulation 2015;132:S444-64.

135 Neal JM, Neal EJ, Weinberg GL. American Society of regional anesthesia and pain medicine local anesthetic systemic toxicity checklist: 2020 version. Reg Anesth Pain Med 2021:46:81-2.

136 Ryu HY, Kim J-Y, Lim HK, et al. Bupivacaine induced cardiac toxicity mimicking an acute non-ST segment elevation myocardial infarction. Yonsei Med J 2007:48:331-6.

137 Cotileas $\mathrm{P}$, Myrianthefs $\mathrm{P}$, Haralambakis A, et al. Bupivacaine-Induced myocardial depression and pulmonary edema: a case report. J Electrocardiol 2000;33:291-6.
138 Baumgartner $\mathrm{H}$, Hung J, Bermejo J, et al. Echocardiographic assessment of valve stenosis: EAE/ASE recommendations for clinical practice. Journal of the American Society of Echocardiography 2009;22:1-23.

139 Parvizi J, Smith EB, Pulido L. The rise in the incidence of pulmonary embolus after joint arthroplasty: is modern imaging to blame? Clinical Orthopaedics and Related Research 2007:463:107-13.

$140 \mathrm{KM} \mathrm{H}$, Burrell M, Rao S. Incidence and risk factors for fatal pulmonary embolism after major trauma: a nested cohort study. British Journal of Anaesthesia 2010;105:596-602.

141 Kasper W, Konstantinides S, Geibel A, et al. Prognostic significance of right ventricular afterload stress detected by echocardiography in patients with clinically suspected pulmonary embolism. Heart 1997;77:346-9.

142 Serafini O, Bisignani G, Greco F. The role of 2D-doppler electrocardiography in the early diagnosis of massive acute pulmonary embolism and therapeutic monitoring] Giornale italiano di cardiologia 1997;27:462-9 http://www.ncbi.nlm.nih.gov/ pubmed/9244751

143 Moore CL, Copel JA. Point-Of-Care ultrasonography. New England Journal of Medicine 2011;364:749-57.

144 Narula J, Chandrashekhar Y, Braunwald E. Time to add a fifth Pillar to bedside physical examination: inspection, palpation, percussion, auscultation, and Insonation. JAMA Cardiol 2018;3:346-50.

145 Boretsky KR, Kantor DB, DiNardo JA, et al. Focused cardiac ultrasound in the pediatric perioperative setting. Anesthesia \& Analgesia 2019:129:925-32.

146 Adler AC, Brown KA, Conlin FT, et al. Cardiac and lung point-of-care ultrasound in pediatric anesthesia and critical care medicine: uses, pitfalls, and future directions to optimize pediatric care. Pediatric Anesthesia 2019;44:790-8.

147 v FM, Stoller JZ, Glau CL. Seeing is believing: ultrasound in pediatric procedural performance. Pediatrics 2019;144.

148 Gagey A-C, de Queiroz Siqueira M, Desgranges F-P, et al. Ultrasound assessment of the gastric contents for the guidance of the anaesthetic strategy in infants with hypertrophic pyloric stenosis: a prospective cohort study. Br J Anaesth 2016;116:649-54.

149 Schmitz A, Thomas S, Melanie F, et al. Ultrasonographic gastric antral area and gastric contents volume in children. Pediatric Anesthesia 2012;22:144-9

150 Marciniak B, Fayoux P, Hébrard A, et al. Airway management in children: ultrasonography assessment of tracheal intubation in real time? Anesthesia \& Analgesia 2009:108:461-5.

151 Alonso Quintela P, Oulego Erroz I, Mora Matilla M, et al. [Usefulness of bedside ultrasound compared to capnography and X-ray for tracheal intubation]. An Pediatr 2014;81:283-8

152 Boretsky KR. Images in anesthesiology: point-of-care ultrasound to diagnose esophageal intubation. Anesthesiology 2018;129:190.

153 Trinavarat P, Riccabona M. Potential of ultrasound in the pediatric chest. Eur J Radiol 2014:83:1507-18.

154 Song I-K, Kim E-H, Lee J-H, et al. Utility of perioperative lung ultrasound in pediatric cardiac surgery: a randomized controlled trial. Anesthesiology 2018;128:718-27.

155 Murphy M, Walls R. Manual of emergency airway management. 4 ed. Philadelphia PA: Wolters Kluwer Health, 2012.

156 Bhananker SM, Ramamoorthy C, Geiduschek JM, et al. Anesthesia-related cardiac arrest in children: update from the pediatric perioperative cardiac arrest registry. Anesthesia \& Analgesia 2007;105:344-50.

157 Hoffmann B, Gullett J, Hill H, et al. Bedside ultrasound of the neck confirms endotracheal tube position in emergency Intubations. Ultraschall in Med 2014;35:451-8

158 Lahham S, Baydoun J, Bailey J, et al. A prospective evaluation of transverse tracheal sonography during emergent intubation by emergency medicine resident physicians. J Ultrasound Med 2017:36:2079-85.

159 Hatch LD, Grubb PH, Lea AS, et al. Endotracheal intubation in neonates: a prospective study of adverse safety events in 162 infants. J Pediatr 2016;168:62-6.

160 EL-Nawawy AA, Abdelmohsen AM, Hassouna HM. Role of echocardiography in reducing shock reversal time in pediatric septic shock: a randomized controlled trial. J Pediatr 2018;94:31-9.

161 Kutty S, Attebery JE, Yeager EM, et al. Transthoracic echocardiography in pediatric intensive care: impact on medical and surgical management. Pediatr Crit Care Med 2014:15:329-35

162 Ranjit S, Aram G, Kissoon N, et al. Multimodal monitoring for hemodynamic categorization and management of pediatric septic shock. Pediatric Critical Care Medicine 2014;15:e17-26.

163 Lai WW, Geva T, Shirali GS, et al. Guidelines and standards for performance of a pediatric echocardiogram: a report from the task force of the pediatric Council of the American Society of echocardiography. Journal of the American Society of Echocardiography 2006;19:1413-30.

164 Marin JR, Lewiss RE, Shook JE, WORLD INTERACTIVE NETWORK FOCUSED ON CRITICAL ULTRASOUND. Point-Of-Care ultrasonography by pediatric emergency medicine physicians. Pediatrics 2015;135:e1113-22. 
165 Riera A, Weeks B, Emerson BL, et al. Evaluation of a focused cardiac ultrasound protocol in a pediatric emergency department. Pediatr Emerg Care 2018; Publish Ahead of Print.

166 Kleinman ME, de Caen AR, Chameides L, et al. Part 10: pediatric basic and advanced life support: 2010 international consensus on cardiopulmonary resuscitation and emergency cardiovascular care science with treatment recommendations. Circulation 2010;122:\$466-515.

167 Steiger HV, Rimbach K, Müller E, et al. Focused emergency echocardiography: lifesaving tool for a 14-year-old girl suffering out-of-hospital pulseless electrical activity arrest because of cardiac tamponade. Eur J Emerg Med 2009;16:103-5.

168 Tsung JW, Blaivas M. Feasibility of correlating the pulse check with focused pointof-care echocardiography during pediatric cardiac arrest: a case series. Resuscitation 2008;77:264-9.

169 Hernandez C, Shuler K, Hannan H, et al. C.A.U.S.E.: Cardiac arrest ultra-sound exam-a better approach to managing patients in primary non-arrhythmogenic cardiac arrest. Resuscitation 2008;76:198-206.

170 Varriale $\mathrm{P}$, Maldonado JM. Echocardiographic observations during in hospital cardiopulmonary resuscitation. Crit Care Med 1997;25:1717-20.

171 Habre W, Disma N, Virag K, et al. Incidence of severe critical events in paediatric anaesthesia (apricot): a prospective multicentre observational study in 261 hospitals in Europe. Lancet Respir Med 2017;5:412-25.

172 Lasa JJ, Rogers RS, Localio R, et al. Extracorporeal cardiopulmonary resuscitation (E(PR) during pediatric in-hospital cardiopulmonary arrest is associated with improved survival to discharge. Circulation 2016;133:165-76.

173 Nasr VG, Gottlieb EA, Adler AC, et al. Selected 2018 highlights in congenital cardiac anesthesia. J Cardiothorac Vasc Anesth 2019;33:2833-42.

174 Saxhaug L. A Web of Science search for publications on "handheld ultrasound," "hand-carried ultrasound" and "pocket-sized ultrasound", formulated 1/11/19.
175 . Available: https://www.itnonline.com/content/mount-sinai-school-medicine-givespocket-ultrasound-devices-its-students [Accessed 10/2/20].

176 . Available: https://www.mobihealthnews.com/news/north-america/uc-irvinemedical-school-gifts-butterfly-handheld-ultrasounds-its-whole-class [Accessed 3/11/19].

177 Mulvagh SL, Bhagra A, Nelson BP, et al. Handheld Ultrasound Devices and the Training Conundrum: How to Get to "Seeing Is Believing". Journal of the American Society of Echocardiography 2014;27:310-3.

178 Kim EY, Park KH, Choi SJ, et al. Educational value of pocket-sized ultrasound devices to improve understanding of ultrasound examination principles and sonographic anatomy for medical student. PLoS One 2017;12:e0185031.

179 Swamy M, Searle RF. Anatomy teaching with portable ultrasound to medical students. BMC Med Educ 2012;12:99.

180 Portable ultrasound improves patient understanding, quality of care. Available: https://www.beckershospitalreview.com/quality/portable-ultrasound-improvespatient-understanding-quality-of-care.html [Accessed 10/10/20].

181 Atkinson PR, Beckett N, French J, et al. Does point-of-care ultrasound use impact resuscitation length, rates of intervention, and clinical outcomes during cardiac arrest? A study from the sonography in hypotension and cardiac arrest in the emergency department (SHoC-ED) Investigators. Cureus 2019;11:e4456.

182 Huis In 't Veld MA, Allison MG, Bostick DS, et al. Ultrasound use during cardiopulmonary resuscitation is associated with delays in chest compressions. Resuscitation 2017;119:95-8.

183 Alzahrani SA, Al-Salamah MA, Al-Madani WH, et al. Systematic review and metaanalysis for the use of ultrasound versus radiology in diagnosing of pneumonia. Crit Ultrasound J 2017:9:6. 